\title{
Evidence for a Supermassive Black Hole in the So Galaxy NGC $3245^{1}$
}

\author{
Aaron J. Barth ${ }^{2}$, Marc Sarzi ${ }^{3,4}$, Hans-Walter Rix ${ }^{4}$, Luis C. Ho ${ }^{5}$, Alexei V. Filippenko ${ }^{6}$, and \\ Wallace L. W. Sargent ${ }^{7}$
}

\begin{abstract}
The S0 galaxy NGC 3245 contains a circumnuclear disk of ionized gas and dust with a radius of 1".1 (110 pc), making it an ideal target for dynamical studies with the Hubble Space Telescope (HST). We have obtained spectra of the nuclear disk with the Space Telescope Imaging Spectrograph, using an 0!'2-wide slit at five parallel positions. Measurements of the $\mathrm{H} \alpha$ and [N II] emission lines are used to map out the kinematic structure of the disk in unprecedented detail. The data reveal a rotational velocity field with a steep velocity gradient across the innermost 0 ". 4 . We construct dynamical models for a thin gas disk in circular rotation, using HST optical images to map out the gravitational potential due to stars. Our modeling code includes the blurring due to the telescope point-spread function and the nonzero slit width, as well as the instrumental shift in measured wavelength for light entering the slit off-center, so as to simulate the data as closely as possible. The $\mathrm{H} \alpha+[\mathrm{N}$ II] surface brightness measured from an $H S T$ narrow-band image is folded into the models, and we demonstrate that many of the apparent small-scale irregularities in the observed velocity curves are the result of the patchy distribution of emission-line surface brightness. Over most of the disk, the models are able to fit the observed radial velocity curves closely, although there are localized regions within the disk which appear to be kinematically disturbed relative to the overall rotational pattern. The velocity dispersion of [N II] $\lambda 6584$ rises from $\sigma \approx 50$ $\mathrm{km} \mathrm{s}^{-1}$ in the outer disk to $\sim 160 \mathrm{~km} \mathrm{~s}^{-1}$ at the nucleus, and most of this linewidth cannot be attributed to rotational or instrumental broadening. To account for the possible dynamical effect of the intrinsic velocity dispersion in the gas, we also calculate models which include a correction for asymmetric drift. This correction increases the derived black hole mass by $10 \%$, but leads to slightly poorer fits to the data. A central
\end{abstract}

\footnotetext{
${ }^{1}$ Based on observations with the NASA/ESA Hubble Space Telescope obtained at STScI, which is operated by AURA, Inc., under NASA contract NAS5-26555.

${ }^{2}$ Harvard-Smithsonian Center for Astrophysics, 60 Garden Street, Cambridge, MA 02138

${ }^{3}$ Dipartimento di Astronomia, Università di Padova, Vicolo dell'Osservatorio 5, I-35122, Italy

${ }^{4}$ Max-Planck-Institut für Astronomie, Königstuhl 17, Heidelberg D-69117, Germany

${ }^{5}$ The Observatories of the Carnegie Institution of Washington, 813 Santa Barbara Street, Pasadena, CA 91101

${ }^{6}$ Department of Astronomy, University of California, Berkeley, CA 94720-3411

${ }^{7}$ Palomar Observatory, 105-24 Caltech, Pasadena, CA 91125
} 
dark mass of $(2.1 \pm 0.5) \times 10^{8} \mathrm{M}_{\odot}$ is required for the models to reproduce the steep central velocity gradient. This value for the central mass is consistent with recently discovered correlations between black-hole mass and bulge velocity dispersion.

Subject headings: galaxies: elliptical and lenticular — galaxies: individual (NGC 3245)

— galaxies: kinematics and dynamics — galaxies: nuclei

\section{Introduction}

During the past few years, tremendous progress has been made in the quest to detect supermassive black holes (BHs) in galactic nuclei. Over 30 dynamical measurements of $\mathrm{BH}$ masses have now been performed, most of them based on data from the Hubble Space Telescope (HST) (for reviews, see Kormendy \& Richstone 1995; Richstone et al. 1998; Ho 1999; Kormendy 2001). These measurements have confirmed and extended early suggestions (Kormendy \& Richstone 1995) that $\mathrm{BH}$ mass is correlated with the mass of the bulge component of the host galaxy, albeit with substantial scatter. An intriguing recent development has been the discovery that the BH mass and bulge velocity dispersion are very tightly correlated (Ferrarese \& Merritt 2000; Gebhardt et al. 2000; Merritt \& Ferrarese 2001). Models of bulge formation and the growth of "seed" BHs and quasar engines have provided theoretical frameworks for understanding the origin of this correlation (Silk \& Rees 1998; Ostriker 2000; Haehnelt \& Kauffmann 2001; Adams, Graff, \& Richstone 2001).

One issue remaining to be resolved is the exact slope of the correlation between $\mathrm{BH}$ mass and stellar velocity dispersion. Gebhardt et al. (2000) and Ferrarese \& Merritt (2000) find different values for the slope, and the various theoretical scenarios make different predictions for this quantity as well. To better understand BH demographics, more mass measurements are needed, and with the highest precision possible. Several HST surveys currently in progress will provide a wealth of new data on BH masses, using both stellar-dynamical and gas-dynamical measurement techniques.

Measurements of ionized gas kinematics in the environment of a $\mathrm{BH}$ can provide the most straightforward means to determine the central mass, provided that the gas is in circular rotation. Using HST spectroscopy, the gas-dynamical method has successfully been applied to several earlytype galaxies and a few spirals. A handful of objects were observed with the HST Faint Object Spectrograph (FOS) (Harms et al. 1994; Ferrarese, Ford, \& Jaffe 1996; van der Marel \& van den Bosch 1998; Ferrarese \& Ford 1999; Verdoes Kleijn et al. 2000). As a single-aperture spectrograph, however, the FOS was an inefficient instrument for mapping velocity fields. The long-slit mode of the Faint Object Camera (FOC) was used successfully in one case, to measure gas kinematics in the nucleus of M87 (Macchetto et al. 1997). Since the installation of the Space Telescope Imaging Spectrograph (STIS), a long-slit spectrograph, it has become possible to map out the velocity structure of ionized gas disks very efficiently, as demonstrated by Bower et al. (1998), Shields et al. (2000), and Sarzi et al. (2001). 
STIS observations provide significant advantages in comparison with the previous generation of HST spectrographs, and BH masses can consequently be measured with greater precision. The ability to measure gas velocities at many positions simultaneously means that the assumption of circular rotation can be tested much more thoroughly, and the disk orientation can be constrained with kinematic data. At the same time, the improvement in data quality also makes it possible to perform more detailed and accurate modeling of the observations, which in turn leads to better constraints on the BH mass. Recent studies have also begun to examine the nuclear disk structure in more detail, including the possibility that asymmetric drift may affect the disk dynamics (Verdoes Kleijn et al. 2000).

This paper presents an analysis of HST observations of NGC 3245, as part of a STIS program designed to study the dynamics of ionized gas in nearby, low-luminosity active galactic nuclei (AGNs). NGC 3245 is an S0 galaxy with a mildly active nucleus classified as a LINER/H II "transition" type (Ho, Filippenko, \& Sargent 1997a). Ground-based spectra displayed by Ho, Filippenko, \& Sargent (1995) show double-peaked profiles of $\mathrm{H} \alpha$ and [N II] $\lambda 6584$, indicating spatially unresolved, rapid rotation near the nucleus. VLA observations reveal an unresolved nuclear radio source, with no apparent jet (Wrobel \& Heeschen 1991). NGC 3245 lies at a distance modulus of $31.60 \pm 0.20$ (a distance of $20.9 \mathrm{Mpc}$ ), as determined by measurements of surface-brightness fluctuations (Tonry et al. 2001).

The HST observations, the basic measurements, and the deprojection of the galaxy's surfacebrightness profile are described in $\S 2$ and $\S 3$. In $\S 4$ we give a detailed description of the technique used to model the velocity field, and $\S 5$ describes the results of this analysis. In $\S 6$ we discuss this work in the context of other $\mathrm{BH}$ mass measurements and the correlations with host galaxy properties.

\section{WFPC2 Imaging}

\subsection{Observations}

Observations of NGC 3245 were obtained with the HST Wide Field and Planetary Camera 2 (WFPC2) through the F658N (narrow-band $\mathrm{H} \alpha+[\mathrm{N} \mathrm{II}]$ ) and $\mathrm{F} 702 \mathrm{~W}$ ( $R$-band) filters, with the nucleus placed on the PC detector (pixel size $0{ }^{\prime \prime} 046$ ). In addition, an archival image through the F547M ( $V$-band) filter was available. The total exposure times were 360, 2200, and $140 \mathrm{~s}$ for F547M, F658N, and F702W, respectively. The F547M and F658N images were each taken as sequences of two individual exposures, and the frames for each filter were combined with rejection of cosmic rays using the STSDAS task CRREJ. Cosmic rays were removed from the single F702W image by clipping deviant pixels using the IRAF task COSMICRAYS. ${ }^{8}$ A continuum-subtracted

\footnotetext{
${ }^{8}$ IRAF is distributed by the National Optical Astronomy Observatories, which are operated by the Association of Universities for Research in Astronomy, Inc., under cooperative agreement with the National Science Foundation.
} 
$\mathrm{H} \alpha+[\mathrm{N}$ II] image was created by converting the F658N and F702W images to flux units using the HST photometric zeropoints and subtracting the scaled F702W flux from the F658N image. The nuclear disk is seen in dust absorption in the $V$ and $R$ images, with an overall appearance very similar to the dusty disks seen in some radio-loud ellipticals such as NGC 4261 (Jaffe et al. 1993) and NGC 7052 (van der Marel \& van den Bosch 1998). The disk is clearly visible in the $\mathrm{H} \alpha+[\mathrm{N} \mathrm{II}]$ image as well, with a bright, compact core of emission surrounded by an outer ring with a major axis radius of 1 ". 1 (Figure 1 ).

From visual inspection of the images, the disk has major and minor axes of projected size $1^{\prime \prime} 1$ and $0^{\prime \prime} 4$, corresponding to a radius of $110 \mathrm{pc}$ and an inclination of $\sim 65^{\circ}$ to the line of sight under the assumption that the disk is intrinsically circular. Due to the "fuzzy" morphology of the disk edges, the inclination cannot be determined precisely from the imaging data alone. The disk major axis and the outer isophotes of the host galaxy are aligned to within a few degrees and are oriented approximately north-south, at position angle (P.A.) $\approx 0^{\circ}$.

The F547M and F702W images were used to create a $V-R$ color map, displayed in Figure 1. This image shows that the dust extinction primarily affects the western half of the disk. Filaments of dust are seen extending southward from the disk to distances of $\sim 3^{\prime \prime}$ from the nucleus. The brightest arcs of $\mathrm{H} \alpha$ emission at the edge of the disk coincide with regions which are bluer than the surrounding starlight in $V-R$, which suggests that recent star formation could be responsible for the $\mathrm{H} \alpha+[\mathrm{N} \mathrm{II}]$ emission in the outer regions of the disk.

\subsection{Stellar Density Profile}

To investigate the central mass concentration in NGC 3245, it is first necessary to determine the stellar mass density by deprojecting the observed surface brightness distribution. The galaxy's surface brightness appears to be affected by dust absorption within the inner $\sim 2^{\prime \prime}$, particularly on the western side of the galaxy. We used the F702W data to determine the stellar light profile since it is less sensitive to dust absorption than the F547M image.

As a first step, we removed the small contribution of emission-line light from the F702W image, by subtracting a scaled copy of the continuum-subtracted $\mathrm{H} \alpha+[\mathrm{N} \mathrm{II}]$ image from the F702W frame, accounting for the exposure time, photometric zeropoint, and passband difference between the two filters. The emission-line contribution to the total count level in the $\mathrm{F} 702 \mathrm{~W}$ frame is highest in the ring of emission at the outer edge of the disk. In this region, the emission lines contribute $3-4 \%$ of the total F702W count level; in other portions of the disk where the $\mathrm{H} \alpha+[\mathrm{N} \mathrm{II}]$ lines have lower equivalent widths the contamination is at the level of $1-2 \%$. At most locations in the disk, however, the [N II] $\lambda 6584$ line falls outside the passband of the F658N filter. This leaves

a small residual contamination of emission-line light in the corrected F702W image. (This effect is somewhat mitigated by the fact that the highest equivalent widths are found on the northern, blueshifted side of the disk, where [N II] is partially within the F658N band.) Also, the [S II] 
$\lambda \lambda 6716,6731$ emission remains as a small contribution to the F702W image. (The [O I] $\lambda \lambda 6300$, 6363 lines are much fainter than [S II] and we ignore their contribution to the F702W image.) We estimated the magnitude of this residual contamination by extracting spectra from our STIS data and using the F702W transmission curve to determine contribution of the $[\mathrm{N} \mathrm{II}]$ and $[\mathrm{S}$ II] lines to the F702W image at different locations in the disk. At most locations in the disk, the residual emission-line contamination is $\lesssim 0.7 \%$ of the total F702W countrate. Our spectra do not have the necessary spatial resolution to perform an exact correction for this emission, but it is sufficiently small that it has a negligible effect on the derived stellar density profile. In any case, the extinction within the nuclear disk is large enough that the uncertainty in the extinction correction (described below) is much greater than the residual emission-line contamination.

We attempted to correct the data for the effects of dust absorption using the technique described by Carollo et al. (1997), using the $V-R$ color as an indicator of dusty regions. This method did remove patchy dust features to some extent, but having only the $V$ and $R$ bands did not provide a sufficient wavelength baseline for the method to be optimal. Instead, we adopted a simpler approach to deal with the extinction. Using the $V-R$ image, we created a map of $E(V-R)$, and from it a map of $A_{R}$, under the assumption that the observed color gradient is entirely due to dust rather than a stellar population gradient. Then, assuming a uniform dust screen, we used the map of $A_{R}$ to correct the $R$-band image for extinction. In the dusty patch just west of the nucleus, the inferred value of $A_{R}$ is $\sim 0.7-0.9$ mag. The dust disk is still visible in the corrected image, but is much less optically thick than in the original frame.

Surface photometry was performed on the extinction-corrected image after masking out the remaining patches of dust located west and south of the nucleus. The surface-brightness profile was measured by fitting ellipses to the isophotes of this image, using the IRAF task ELLIPSE. No rotations or other transformations which could potentially blur the nuclear light distribution were performed prior to fitting isophotes. Within the inner $r=1^{\prime \prime}$, the isophotes of the masked image are nearly circular, with $\epsilon \approx 0.1$ (Figure 2). This enabled us to treat the surface brightness as circularly symmetric, and the stellar distribution as spherically symmetric, at least within the radial range where our kinematic measurements for the ionized gas probe the galaxy potential.

The stellar contribution to the gravitational potential was measured by modeling the surfacebrightness profile as a sum of Gaussian components, using the method described by Monnet, Bacon, \& Emsellem (1992) and Emsellem et al. (1994). A major advantage of the multi-Gaussian method is that it leads to a simple reconstruction of the intrinsic surface brightness distribution, provided that the telescope point-spread function (PSF) can be approximated as a sum of Gaussian components. The multi-Gaussian method also leads to a straightforward deprojection of the surface brightness $\Sigma(r)$ into the intrinsic luminosity density distribution $\rho(r)$, which also can be expressed as the sum of a set of Gaussians. Then, assuming a spatially constant mass-to-light ratio $\Upsilon$, we obtain a multi-Gaussian description for the stellar mass density as the sum of spherical mass components having a potential $\Phi_{\star}$ that can be easily computed in terms of error functions. Additional examples of the method, and its application to HST images, are described by Sarzi et al. (2001). In the 
fits, we used a multi-Gaussian description for a synthetic F702W PSF generated by the Tiny Tim program (Krist \& Hook 1999).

The F702W count rates were converted into absolute $R$-band magnitudes using the photometric zeropoints given by Holtzman et al. (1995) and adopting the $V-R$ color within $r=8^{\prime \prime}$ given by Michard \& Poulain (2000). For the conversion between surface brightness and mass, we also corrected for Galactic extinction using the dust map of Schlegel, Finkbeiner, \& Davis (1998) combined with the reddening curve of Cardelli, Clayton, \& Mathis (1989). The Galactic $R$-band extinction toward NGC 3245 is $A_{R}=0.067$ mag.

We performed a decomposition of the stellar profile into two components: the spatially extended starlight, and a central, unresolved source. From the decomposition, we find that the nuclear point source has $M_{R}=-12.3 \mathrm{mag}$. We assume that this component represents the nonstellar continuum of the weakly active nucleus. The deprojection of the stellar profile into a three-dimensional luminosity density was performed after subtracting the pointlike nuclear component from the total profile.

The fit to the observed surface brightness $\Sigma(r)$, the deprojected luminosity density profile $\rho(r)$, and the corresponding circular velocities for $\Upsilon=1$ (in $R$-band solar units) are shown in Figure 3. Since the extinction correction is derived from the ratio of the $V$ and $R$ images, the dust-corrected image is extremely noisy, particularly in the innermost pixels. As a result, it is not possible to accurately measure the cusp slope in the inner $r \approx 2$ pixels (or $\sim 10 \mathrm{pc}$ ). The deprojected stellar density profile has a broad bump in the inner $4 \mathrm{pc}$ which cannot be entirely attributed to the nuclear point source. This is an unavoidable problem because of the large extinction correction. However, the uncertainty in the central density slope ultimately has a very small effect on the dynamical models because at these small radii the gravitational potential is dominated by the $\mathrm{BH}$.

The mass of the nuclear disk itself can be estimated from the reddening observed in the $V-R$ image. Following Ferrarese \& Ford (1999), we estimate the dust mass per unit surface area in

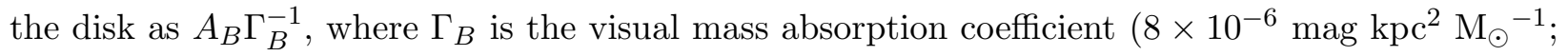
Sadler \& Gerhard 1985). To estimate the maximum possible mass of the disk, we use the reddening derived from the dusty southwestern quadrant of the disk. For a mean reddening of $E(V-R)=$ $0.18 \mathrm{mag}$, we obtain $A_{B}=0.93 \mathrm{mag}$ and a dust mass of $1.9 \times 10^{4} \mathrm{M}_{\odot}$ for the disk. Then, applying a Galactic gas-to-dust mass ratio of $1.3 \times 10^{2}$, the total disk mass is $2.4 \times 10^{6} \mathrm{M}_{\odot}$. This is small enough to be safely neglected in the dynamical calculations. The mass of the ionized gas in the disk can be estimated from the $\mathrm{H} \alpha$ luminosity and the density derived from the [S II] lines (e.g., Osterbrock 1989). Using the values of $n_{e}$ and $L(\mathrm{H} \alpha)$ from Ho, Filippenko, \& Sargent (1997a), we obtain a mass of $\sim 5 \times 10^{4} \mathrm{M}_{\odot}$ for the ionized gas in the disk.

The mass of warm ( $\gtrsim 20 \mathrm{~K}$ ) dust in the galaxy can be calculated using IRAS fluxes (Young et al. 1989; Goudfrooij \& de Jong 1995). Following the method described by Young et al. (1989), we find that the total warm dust mass in NGC 3245 is $\sim 2.5 \times 10^{5} \mathrm{M}_{\odot}$. This is similar to the dust masses estimated for other early-type galaxies such as NGC 4261 (e.g., Martel et al. 2000). 
However, the mass derived by this method applies to the galaxy as a whole, rather than just the nuclear disk, because of the large beamsize of the IRAS observations.

\section{STIS Spectroscopy}

\subsection{Observations}

Long-slit spectra were obtained with STIS using the 0"'2-wide slit and the G750M grating, which gives a point-source spectral resolution of FWHM $\approx 0.9 \AA$. The STIS CCD was read out in unbinned mode, with a pixel scale of $0.554 \AA$ along the dispersion direction and $0^{\prime \prime} 0507$ along the spatial axis in the final calibrated image. The grating tilt was set to give wavelength coverage over 6300-6860 A. Prior to taking the spectroscopic exposures, target acquisition and peak-up were performed to center the spectrograph slit on the nucleus.

Following the peak-up, the slit was offset by 0.5 to the southeast, and spectroscopic images were taken at five parallel slit positions with a gap of $0{ }^{\prime \prime} 05$ between adjacent positions and with the third exposure at the location of the peak-up. The exposure times for each position are listed

in Table 1. Figure 1 shows the slit locations overplotted on the WFPC2 F702W image. The spectrograph was oriented with the slit at P.A. $=202^{\circ}$, in the sense that the top of the slit (the instrumental $+y$ direction) was rotated $202^{\circ}$ eastward from celestial north (see Figure 1). This orientation is offset by approximately $28^{\circ}$ from the major axis of the galactic isophotes.

Observations of internal line lamps were obtained during each orbit for wavelength calibration. The wavelength scale was found to shift by $0.02-0.05 \AA$ between successive calibration frames. The small shifts of the wavelength scale during the course of each orbit can be neglected, as they are much smaller than the measurement uncertainties in the velocity centroids.

At each slit position, three individual exposures were taken for purposes of cosmic-ray removal. Six total orbits were allocated for the five slit positions. We attempted to balance the exposure times between the five slit positions within the constraints of a predefined HST offsetting pattern, but the use of the predefined pattern resulted in a substantially longer exposure for the fifth slit position. The data were processed by the standard STIS calibration pipeline, which includes bias subtraction, flat-fielding, cosmic-ray removal and stacking of individual exposures, and wavelength and flux calibration. The pipeline calibration also corrects the wavelength scale to the heliocentric reference frame. The final step of the calibration pipeline is a geometric correction that spatially rectifies the image to a linear plate scale in the spatial and dispersion directions. Before applying the geometric correction, we performed an additional step of cleaning residual hot pixels and cosmicray hits from the flux-calibrated images. The central portion of each spectral image is displayed in Figure 4; the starlight continuum has been subtracted from the data so that the emission-line structure is clearly visible at the nucleus. Figure 5 shows an $00^{\prime \prime} 25$-wide extraction of the nuclear spectrum from slit position 3 . 


\subsection{Measurement of Emission Lines}

The emission-line velocities, linewidths, and fluxes were measured from the STIS images by fitting a triplet of Gaussians to the $\mathrm{H} \alpha+[\mathrm{N}$ II] emission blend at each position. Individual rows were extracted out to a distance of 1".5 from the slit center. At larger distances the emission-line brightness dropped off rapidly, as expected from the disk structure seen in the WFPC2 H $\alpha+[\mathrm{N}$ II] image. The profile fits were performed independently on each single-row extraction. Prior to fitting, the underlying starlight was subtracted in a rudimentary way by fitting a straight line to the continuum regions $6520-6540 \AA$ and $6660-6680 \AA$, and subtracting the fitted continuum from the spectrum. Over this wavelength range, a straight line gives an adequate fit to the continuum shape.

In the fits, the [N II] $\lambda \lambda 6548,6584$ lines were constrained to have the same FWHM in velocity units and a flux ratio of 2.96:1. All three lines were constrained to have the same mean velocity, but the $\mathrm{H} \alpha$ and $[\mathrm{N} \mathrm{II}]$ lines were allowed to have different widths. For the central slit at positions within \pm 1 row of the nucleus, the $\mathrm{H} \alpha+[\mathrm{N}$ II] blend revealed a broad base of emission, and a Gaussian broad $\mathrm{H} \alpha$ component was added to improve the fits. ${ }^{9}$ Over most of the disk, the Gaussian model provided a reasonable fit to the line profiles within the uncertainties. At some locations the lines were more sharply peaked than a Gaussian, but the fits still generally resulted in $\chi^{2}$ per degree of freedom $\left(\chi^{2} / d\right)$ near unity. The lines [O I] $\lambda \lambda 6300,6363$ and [S II] $\lambda \lambda 6716,6731$ are visible as well, but at much fainter levels. Little kinematic information could be derived from these lines, and our analysis is based exclusively on the measurements of $\mathrm{H} \alpha$ and [N II].

At many locations, the redshifts of $\mathrm{H} \alpha$ and [N II] were discrepant by an amount larger than the formal uncertainties in the line velocities derived from profile fits. This is one indication that there may be at least two kinematically distinct populations of clouds within the disk; the two emission lines have systematically different widths as well (§4.5). The fitting technique described above assumes that $\mathrm{H} \alpha$ and $[\mathrm{N} \mathrm{II}]$ share the same redshift, so the measurements essentially give a weighted mean of the individual $\mathrm{H} \alpha$ and $[\mathrm{N} \mathrm{II}]$ line velocities as the best estimate of the projected line-of-sight rotation velocity.

Figure 6 displays the radial velocity curves measured from the $\mathrm{H} \alpha+[\mathrm{N} \mathrm{II}]$ lines. While there is a steep velocity gradient across the innermost 0.'4 of slit position 3, we do not detect a Keplerian rise in velocity in the innermost regions. The steep but smooth central velocity gradient is the result of the blurring of the intrinsic velocity field by the telescope PSF, as well as the blending of light from different locations in the disk within the 0 ' 2 -wide aperture. To derive an accurate $\mathrm{BH}$ mass, it is necessary to model the influence of the telescope and instrument optics ( $\S 4)$, so that the data can be adequately compared with dynamical models for the rotating disk.

Inspection of the 2-dimensional spectra reveals a gradient in the intensity ratio of $\mathrm{H} \alpha$ to $[\mathrm{N}$ II]

\footnotetext{
${ }^{9}$ Ho et al. (1997b) list NGC 3245 as having an "ambiguous" detection of broad H $\alpha$, from their ground-based spectra.
} 
across the disk. For example, Figure 4 shows that in slit position $3, \mathrm{H} \alpha$ is more prominent than $[\mathrm{N} \mathrm{II}]$ in the outer disk while [N II] is stronger relative to $\mathrm{H} \alpha$ at the nucleus. The $[\mathrm{N} \mathrm{II}] \lambda 6584 / \mathrm{H} \alpha$ intensity ratio at each point is shown in Figure 7 (excluding the broad component of $\mathrm{H} \alpha$ at the nucleus). This ratio is relatively constant at $\sim 0.5-0.6$ in the outer disk but rises steeply within $r=0.3$, reaching a peak of 4.5 at the nucleus. Such high $[\mathrm{N} \mathrm{II}] / \mathrm{H} \alpha$ ratios indicate that the ionized gas in the inner disk is excited either by photoionization by a hard (AGN-like) spectrum (e.g., Ho, Filippenko, \& Sargent 1993), or by shock heating, similar to the case of the nuclear disk in M87 (Dopita et al. 1997). In the outer disk, some of the ionized gas may be in H II regions surrounding young, massive stars, and the region of blue continuum emission around the outer rim of the disk further supports this possibility. The [O I] $\lambda 6300$ line is almost undetectable at most locations, consistent with the classification of NGC 3245 as a "transition"-type galaxy with properties intermediate between LINERs and H II nuclei.

\section{Velocity Field Modeling: Method}

\subsection{Basic Steps}

Modeling the observed velocity field proceeds in two steps. First, a model velocity field is generated and projected onto the plane of the sky. Assuming a thin disk in perfect circular rotation, the velocity field is determined by the BH mass $M_{\bullet}$, the stellar density profile and the stellar massto-light ratio $\Upsilon$, and the disk inclination $i$. Transformation to the observed coordinate system, with projected radii in arcseconds, depends on the distance. Next, the model is synthetically "observed" in a way that simulates as closely as possible the actual configuration of the STIS observations. The synthetic observation of the model depends on the angle $\theta$ of offset between the disk major axis and the position angle of the STIS slit, and on the location of each slit position. The BH mass is determined by finding the model parameters which produce the best match to the observed velocity curves. This technique is similar to the methods described by Macchetto et al. (1997), van der Marel \& van den Bosch (1998), and Verdoes Kleijn et al. (2000) for HST FOC and FOS data, and by Bertola et al. (1998) for analysis of ground-based data.

We use cylindrical coordinates $(r, \phi, z)$ for the disk, and assume that the disk is confined to the $(r, \phi)$ plane. The circular velocity $v_{c}$ at a given radius $r$ is determined by $M_{\bullet}$ and by the total stellar mass enclosed by the circular orbit. If the $y^{\prime}$ axis corresponds to the major axis of the disk on the plane of the sky and the origin is taken to be the center of rotation, then the radius at a projected location $\left(x^{\prime}, y^{\prime}\right)$ on the sky plane is given by

$$
r^{2}=\frac{\left(x^{\prime}\right)^{2}}{\cos ^{2} i}+\left(y^{\prime}\right)^{2}
$$

At each point $\left(x^{\prime}, y^{\prime}\right)$ in the model grid, the circular velocity (relative to the systemic velocity $v_{\text {sys }}$ ) 
is given by

$$
v_{c}=\left(\Upsilon v_{\star}^{2}+\frac{G M_{\bullet}}{r}\right)^{\frac{1}{2}},
$$

where $v_{\star}$ is the circular velocity at radius $r$ that would result from the stellar population alone in the absence of a $\mathrm{BH}$, as determined from the profile fits to the WFPC2 data, normalized to a stellar mass-to-light ratio of unity. The mass-to-light ratio $\Upsilon$ includes the contribution of spatially distributed dark matter. We assume that the mass-to-light ratio of the extended massive component (stars plus dark matter) is spatially constant over the spatial scale of the model $(r \leq 100 \mathrm{pc})$.

In the STIS coordinate system, the $y$ axis corresponds to the direction along the slit length and the $x$ axis is the direction of the slit width. The $\left(x^{\prime}, y^{\prime}\right)$ and $(x, y)$ coordinate systems are related by the transformation

$$
\begin{gathered}
x^{\prime}=x \cos \theta-y \sin \theta, \\
y^{\prime}=x \sin \theta+y \cos \theta .
\end{gathered}
$$

\section{2. $\quad$ Model Calculation}

The synthetic observation of the model velocity field for each STIS CCD row is essentially a summation of the intrinsic line-of-sight velocity profiles at every point in the disk, weighted by the emission-line surface brightness of the disk and by the telescope PSF. The 0 '! 2 -wide slit projects to a width of almost exactly four STIS CCD pixels. For a given CCD row $y$, let $\left(x_{0}, y\right)$ be the point in the model velocity field at the left side of the slit. Then, the intersection of the slit aperture and the CCD row will subtend pixels from $x_{0}$ to $x_{1}=x_{0}+3$ in the model velocity field. We assume that the intrinsic line-of-sight velocity profiles are Gaussian prior to passage through the telescope optics. Then, the line profile in velocity units for this slit position and CCD row is given by

$$
f_{y}(v)=\sum_{x=x_{0}}^{x_{1}} \sum_{i} \sum_{j} S_{i j} P(i, j \mid x, y) \exp \left(\frac{\left[v-v_{p}(i, j)-v_{d} M_{a}\left(x-x_{c}\right)\right]^{2}}{-2\left(\sigma_{p}^{2}+\sigma_{\mathrm{th}}^{2}+\sigma_{\mathrm{LSF}}^{2}\right)}\right),
$$

where $S_{i j}$ is the $\mathrm{H} \alpha+[\mathrm{N} \mathrm{II}]$ surface brightness at pixel $(i, j), P(i, j \mid x, y)$ is the value at pixel $(x, y)$ of a PSF centered at pixel $(i, j)$, and $v_{p}(i, j)$ is the projected line-of-sight velocity at $(i, j)$. The quantity $v_{d}$ is the bin size of the CCD pixels along the dispersion axis in the wavelength range of interest, expressed in velocity units $\left(25.2 \mathrm{~km} \mathrm{~s}^{-1}\right), x_{c}$ is the $x$ position of the slit center, and $\left(x-x_{c}\right)$ is in units of pixels. The velocity offset $v_{d} M_{a}\left(x-x_{c}\right)$ is the shift due to the nonzero width of the slit and its projection onto the STIS CCD; it accounts for the fact that the wavelength recorded for a photon depends on the position at which the photon enters the slit along the $x$ axis. The anamorphic magnification factor $M_{a}$ gives the ratio of plate scales in the dispersion and spatial directions. In the wavelength range of interest, $M_{a}=0.93$ for the G750M grating (Bowers $\&$ Baum 1998). The quantity $\sigma_{p}$ is a possible bulk velocity dispersion for gas in the circumnuclear disk, projected along the line of sight, and $\sigma_{\mathrm{th}}$ is the thermal velocity dispersion in the disk. The 
$\mathrm{H} \alpha+\left[\mathrm{N}\right.$ II] surface brightness profile $S$ is discussed in $\S 4.3$, and the derivations of $v_{p}$ and $\sigma_{p}$ are discussed in $\S 4.5$ and $\S 4.6$. The quantity $\sigma_{\mathrm{LSF}}$ is the Gaussian width of the point-source line-spread function (LSF) for STIS (§4.5).

The summations over $i$ and $j$ are assumed to extend over the entire model velocity field, as the extent of the PSF is comparable to the angular size of the NGC 3245 disk. We found, however, that omitting the extended PSF wings, beyond a radius of about 0 ". 4 , had a negligible effect on the model results. Since the computation time increases steeply with increasing PSF size, we performed our model calculations using a subsection of the full PSF with dimensions $0 ! 9 \times 0$. 9 . The omitted flux from the extended PSF wings amounts to only $5 \%$ of the total flux in the PSF.

In practice, the entire procedure described above was calculated on a subsampled pixel grid, with a subsampling factor $s$ ranging from 2 to 6 relative to the STIS pixel scale. Thus, each model subpixel element was $0 . \prime 0507 / s$ on a side. Especially at locations near the nucleus, where there are large gradients both in velocity and in $\mathrm{H} \alpha$ surface brightness, a higher value of $s$ can in principle yield a more accurate model calculation. On a subsampled pixel grid, calculating the line profile for a given CCD row requires a summation over $s$ rows of subpixel elements (i.e., over subsampled rows $y_{0}$ to $y_{1}=y_{0}+s-1$ ). This adds one additional level of summation to equation (4); see Figure 8 for an illustration. In the limit of high values of $s$, this summation converges to an integration over infinitesimal pixels, similar to the expressions given in equation (10) of Macchetto et al. (1997) or equation (1) of Bertola et al. (1998). Since the PSF and the $\mathrm{H} \alpha+[\mathrm{N} \mathrm{II}]$ surface brightness are defined on a discrete pixel grid, the actual calculation that we perform is a summation over pixels rather than an integration of analytic functions.

We used Tiny Tim PSFs for STIS, which were generated on subsampled pixel grids to match the resolution of the model calculations. The PSFs were computed for a monochromatic source at $6600 \AA$. Two distinct physical effects contribute to the PSF: an optical component resulting from diffraction of light through the HST and STIS apertures, and a charge-diffusion component which arises from the bleeding of electrons from each CCD pixel into adjacent pixels. These two components must be treated separately in the modeling process, because the optical PSF will scatter light into the slit from every position within the PSF radius as in equation (4) above, while the charge-diffusion component of the PSF will only cause a spreading of charge from each pixel into its immediate neighbors. The charge-diffusion portion of the PSF accounts for a significant fraction of the total PSF: roughly $20 \%$ of the charge incident on a given pixel will bleed into the adjoining pixels (Krist \& Hook 1999). Thus, the quantity $P$ in equation (4) denotes only the optical component of the PSF and not the charge-diffusion portion, which is applied at a later stage in the model calculation. With the Tiny Tim program, PSFs generated on subsampled pixel grids contain only the optical component by default.

PSFs determined from STIS imaging observations could in principle be used as well, but would suffer from several disadvantages: limited signal-to-noise ratio $(\mathrm{S} / \mathrm{N})$ and dynamic range; a lack of information on the sub-pixel structure of the PSF for subsampled models; and the inability to 
disentangle the optical and charge-bleeding components. In addition, STIS has no narrow-band $\mathrm{H} \alpha$ filter, so any PSF determined from imaging observations would be obtained with either a clear aperture or a long-pass filter, and its structure would differ significantly from the monochromatic PSF appropriate for the spectroscopic data. One drawback of Tiny Tim PSFs for STIS is that they do not include spatial variations in focus aberrations for different positions on the CCD (Krist \& Hook 1999). However, in the NGC 3245 STIS data, $\mathrm{H} \alpha$ falls almost exactly at the center of the CCD, where the Tiny Tim PSF should be most accurate.

The synthetic line profiles were calculated on a velocity grid with a bin size of $25.2 \mathrm{~km} \mathrm{~s}^{-1}$ in order to match the STIS pixel scale in the relevant wavelength range. The coarseness of this wavelength scale has no effect on the derived velocity centroids or the linewidths of the synthetic profiles (to better than $1 \mathrm{~km} \mathrm{~s}^{-1}$ ) because the model profiles are noise-free. For each slit position, the array of model line profiles forms a synthetic 2-dimensional spectrum, analogous to the STIS spectroscopic data and with the same pixel scale (Figure 4). The 2-dimensional model profiles were then convolved with the CCD charge-diffusion kernel to simulate the bleeding of charge between adjacent pixels. This kernel is approximated by a $3 \times 3$ pixel array given by Krist \& Hook (1999) for the STIS CCD. This convolution only results in very small changes (a few $\mathrm{km} \mathrm{s}^{-1}$ ) to the radial velocity curves and linewidths, but it is included for completeness.

Finally, to simulate the measurement of the actual STIS data, each synthetic line profile was measured by extracting individual rows and fitting a Gaussian model, analogous to the measurement of the actual STIS data. The synthetic profiles were nearly Gaussian in shape except within the inner $\sim 0^{\prime \prime} 3$, where the large velocity gradient at the nucleus produced asymmetries and extended low-amplitude wings on the profiles. In principle, it would be simpler and computationally faster to calculate a weighted average velocity for the light entering the slit (for example, see Macchetto et al. 1997), rather than computing a line profile and performing a profile fit. However, we found that for slit positions near the nucleus, the weighted average velocity gave misleading results because of the asymmetric wings on the line profiles. Fitting the calculated profiles with a Gaussian ensures that the data and models are treated identically. Also, computing the full line profile allows a comparison with the observed line widths, in addition to the velocity curves.

One aspect of the data which we do not model is the geometric rectification of the image. This correction spatially resamples the image to rebin it to a linear pixel scale (using bilinear interpolation), and rectifies the image so that the dispersion direction is aligned parallel to the CCD rows. As a result, the spatial and wavelength scales of the corrected image are expanded in some locations and compressed at other locations, relative to the original image. Our model calculation is performed on a spatially rectified grid with a linear pixel scale, and there is no simple way to account for the spatially variable effects of the geometric correction. To check whether the geometric correction affects the linewidths, we measured the width of [N II] from the uncorrected image. There are small differences in the linewidths measured from the corrected and uncorrected images, but overall the geometrically corrected image does not show systematically broader lines, indicating that the geometric correction should not have a significant effect on the derived value of 
$M_{\bullet}$.

\subsection{Emission-Line Surface Brightness}

In the model calculation, the line profiles must be weighted by the emission-line surface brightness at each point in the disk. One option is to use a smooth analytic approximation to the observed surface brightness, similar to the method used in most previous work (e.g., van der Marel \& van den Bosch 1998; Verdoes Kleijn et al. 2000). As an initial step, we parameterize the $\mathrm{H} \alpha+[\mathrm{N}$ II] surface brightness by $S(r)=S_{0}+S_{1} e^{-r / r_{1}}$. (We will refer to this as the exponential surface-brightness model.) The three parameters $S_{0}, S_{1}$, and $r_{1}$ were determined by fitting a model to the inner $r=0.5$ of the disk and optimizing the model fit to the spatial profile of $\mathrm{H} \alpha+[\mathrm{N} \mathrm{II}]$ flux in the STIS data. The results of the fit are shown in Figure 9. We find $S_{0}=1$ and $S_{1}=26$ (in arbitrary units), and $r_{1}=6.3 \mathrm{pc}$; similar results are found by fitting directly to the surface brightness profile of the WFPC2 $\mathrm{H} \alpha+[\mathrm{N} \mathrm{II}]$ image. Folding the exponential surface-brightness profile into the model calculation results in smooth velocity curves, as shown in the left panel of Figure 10.

For NGC 3245, the WFPC2 $\mathrm{H} \alpha+[\mathrm{N}$ II] image provides sufficient $\mathrm{S} / \mathrm{N}$ and spatial coverage that the image itself can be folded into the model calculations, rather than using a smooth model for the surface brightness. Even though [N II] $\lambda 6584$ is mostly outside the F658N filter band, the WFPC2 image can still provide an approximate description of the $\mathrm{H} \alpha+[\mathrm{N}$ II] flux distribution, and it allows the models to incorporate the small-scale structure in the emission-line surface brightness profile. Before performing model calculations with the $\mathrm{H} \alpha+[\mathrm{N}$ II $]$ image, we first removed the contribution of the broad $\mathrm{H} \alpha$ component from the nucleus, because the radial velocity curves were measured only for the narrow components. In the central row extraction of slit position 3 , the broad component of $\mathrm{H} \alpha$ accounts for $46 \%$ of the total emission-line flux that would be measured through the F658N filter at this position. This broad-line contribution was subtracted off from the F658N image by scaling a Tiny Tim PSF image to the appropriate flux and subtracting it from the continuum-subtracted $\mathrm{H} \alpha+[\mathrm{N}$ II] image.

The emission-line map must represent the surface brightness prior to convolution with the telescope optics, since it will effectively be convolved with the STIS PSF in the model calculation. To prepare the $\mathrm{H} \alpha+[\mathrm{N} \mathrm{II}]$ map, we first resampled the WFPC2 image onto a pixel grid subsampled by a factor of $s$ with respect to the original PC pixel size ( $\left.0^{\prime \prime} 046\right)$, and then deconvolved it using the Richardson-Lucy algorithm using the IRAF task LUCY. The deconvolution was performed using a synthetic Tiny Tim WFPC2 PSF generated on a pixel grid subsampled by a factor of $s$. Beyond about 10 iterations, the deconvolution tended to amplify noise peaks to unacceptable levels, and we halted the deconvolution at 10 iterations.

After deconvolution, the image was rotated to match the orientation of the STIS observations, and resampled again to a pixel size which matched the scale of the velocity field map $\left(00^{\prime \prime} 0507 / s\right.$

pixel $^{-1}$ ). The location of the dynamical center was assumed to be coincident with the nuclear peak 
in the $\mathrm{H} \alpha+[\mathrm{N} \mathrm{II}]$ image. The pixel values of this deconvolved image were then used for the surface brightness in the model calculations, as described by equation (4). This method gives a good fit to the $\mathrm{H} \alpha+[\mathrm{N} \mathrm{II}]$ line fluxes measured from the STIS data over the entire face of the disk (Figure 9). We do not attempt to fit the surface-brightness distributions of $\mathrm{H} \alpha$ and [N II] separately, as we do not have sufficient information to disentangle the individual contributions of $\mathrm{H} \alpha$ and [N II] to the WFPC2 image.

By folding the observed surface brightness into the calculations, the models are able to reproduce some of the apparent irregularities in the observed velocity curves. In part, this is due to the velocity gradient in the portion of the disk subtended by the slit. An equally important effect is the instrumental shift in apparent wavelength for light entering the slit off-center. With the 0 '! 2 -wide slit, light entering at the edge of the slit will suffer an instrumental offset of roughly 2 pixels in the $x$ direction, or $\sim 50 \mathrm{~km} \mathrm{~s}^{-1}$, compared to light entering at the slit center. The observed velocity curves show irregular bumps and wiggles at roughly this level, similar to the irregularities seen in the velocity structure of other nuclear disks (e.g., Sarzi et al. 2001).

Figure 10 demonstrates that many (but not all) of the apparent irregularities in the velocity curves are the result of the patchy distribution of emission-line light, rather than being real deviations from circular rotation. This gives additional confidence that the nuclear disk can be modeled successfully in terms of a thin disk in circular rotation. It also allows the identification of localized regions which genuinely deviate from the overall circular velocity pattern.

One additional detail is the treatment of the central subpixel element in the model calculation. The rotation velocity for this central pixel is undefined, and it is located at the peak of the emissionline surface-brightness distribution. We tried two methods to handle this singular point, by setting either the rotation velocity or the emission-line surface brightness of the central pixel to zero. We obtained a better fit to the data by setting the rotation velocity to zero in the central pixel, but the resulting radial-velocity curves were nearly identical in either case. Since our measurements are relatively insensitive to the very high-velocity wings on the line profiles, the treatment of the innermost pixel makes little difference to the model predictions as long as the model is calculated on a sufficiently fine grid.

\subsection{Location of STIS Apertures}

In the modeling, an issue of paramount importance is the determination of the actual positions of the STIS apertures relative to the nucleus of NGC 3245. An error of even half a pixel in the central slit location would have a substantial effect on the model results. (The radial velocity curves for the outer positions are less sensitive to the precise location of the slit.) The observing program was nominally designed to obtain five parallel slit positions with a separation of 0 '.25 between adjacent pointings, and with the third pointing centered at the nucleus. However, the asymmetry

in the radial velocity curve for slit position 3 demonstrates that the center of rotation must have 
been located off-center in the slit.

One constraint on the slit locations comes from the peak-up image, which is an undispersed image of the galaxy as seen through the STIS slit. The peak-up procedure is designed to maximize the amount of light entering the slit, and the asymmetric distribution of dust at the nucleus resulted in the galaxy's nucleus being offset with respect to the slit center. This miscentering is seen clearly in the peak-up image, and from visual inspection of this image we estimate that the brightest point at the nucleus was located $\sim 1.5-2$ pixels from the slit center after the peak-up.

We determined the slit positions quantitatively by two methods, comparing the continuum and emission-line light profiles of the spectroscopic data with the imaging observations. The continuum light profiles in the spectra were compared with profiles measured from the STIS acquisition image, a $5 \mathrm{~s}$ exposure taken with a long-pass filter admitting light in the range 5500-10000 $\AA$. For each of the STIS spectroscopic images, a continuum light profile was calculated by collapsing the spatial profile along the wavelength axis over a wavelength range of 6400-6500 $\AA$. Comparison profiles were created from the acquisition image by extracting 4-pixel-wide strips oriented along the slit direction. The spatial position of each spectroscopic slit was determined by computing a $\chi^{2}$ parameter for the difference between the spectroscopic continuum profile and each comparison profile extracted from the image. This method confirms that the central slit position was miscentered with respect to the brightest pixel at the nucleus, with the best results obtained for an offset between 1 and 2 STIS pixels between the slit $x$ center and the nuclear peak. The derived positions of the other slits are all consistent with the nominal offsets of 0 '.25 perpendicular to the slit length between successive exposures. There is no evidence for any translation of the telescope along the slit direction; the maximum possible shift between exposures is 0.'01-0!'02, or less than 0.5 STIS pixels.

To determine the slit positions with sub-pixel accuracy, we ran a model fit comparing the emission-line flux curves from the STIS spectra (Figure 9) with the model results based on folding the WFPC2 emission-line surface-brightness profile into the model calculation. The separation between adjacent slits was fixed at the nominal value of $0{ }^{\prime \prime} 05$ but the zeropoint of the five slit positions was allowed to float freely in the $x$ and $y$ directions, and the model was calculated with $s=4$. The model fit was optimized by calculating a $\chi^{2}$ parameter for the difference between the measured and modeled emission-line flux curves, as shown in Figure 9. The fit converged on a position for the galaxy nucleus which was 1.5 pixels offset from the slit center in the $x$ direction, in good agreement with the constraints on the slit locations from the continuum profiles and the peak-up image.

Based on these results, we fixed the position of the central slit in the modeling analysis so that the dynamical center of the galaxy in slit position 3 was located 1.5 CCD pixels (0.'076) away from the slit center, as measured along the instrumental $-x$ direction (the direction perpendicular to the slit length). In the $y$ direction, the galaxy's dynamical center was fixed at the center of a STIS pixel, with an uncertainty of $\sim 0.25$ pixel based on the model fits to the emission-line flux distribution. 


\subsection{Linewidths}

In this section we discuss the various effects that contribute to the observed linewidths. There are three major contributions: instrumental broadening, the blending of light from different parts of the disk within the slit aperture (i.e., rotational broadening), and a possible intrinsic velocity dispersion in the disk itself. For the thermal contribution to the line broadening, we use $\sigma_{\mathrm{th}}=10$ $\mathrm{km} \mathrm{s}^{-1}$, as expected for $T \approx 10^{4} \mathrm{~K}$. This contribution is assumed to be isotropic.

The measured velocity dispersion of [N II] (uncorrected for instrumental broadening) increases steadily from $\sim 50 \mathrm{~km} \mathrm{~s}^{-1}$ in the outer disk to $\sim 160 \mathrm{~km} \mathrm{~s}^{-1}$ at the nucleus. $\mathrm{H} \alpha$ is narrower than $[\mathrm{N} \mathrm{II}]$ throughout the disk (Figure 11). The difference between the $\mathrm{H} \alpha$ and $[\mathrm{N} \mathrm{II}]$ linewidths may be partly due to Balmer absorption in the continuum underlying $\mathrm{H} \alpha$. The highest $\mathrm{H} \alpha$ equivalent widths (up to $30 \AA$ ) are found at the northern edge of the disk, and $\mathrm{H} \alpha$ is still systematically narrower than $[\mathrm{N} \mathrm{II}]$ in this region, but the blue continuum in this region may indicate a young stellar population which could contribute additional Balmer absorption. Thus, it is unclear whether $\mathrm{H} \alpha$ and $[\mathrm{N} \mathrm{II}]$ have intrinsically different widths. Unfortunately, [O I] and [S II] are too weak to be useful for a systematic comparison, as done for example by Corsini et al. (1999). Since H $\alpha$ may be affected by underlying absorption, we use the [N II] linewidths as the main comparison for our model calculations.

The instrumental LSF for a point source (including broadening by the telescope PSF and charge diffusion on the CCD) can be approximated by a Gaussian with $\sigma \approx 17 \mathrm{~km} \mathrm{~s}^{-1}$ for the G750M grating at $\lambda=6590 \AA$ (Leitherer et al. 2000). The observed line widths are much broader than this, so it is not necessary to model the detailed structure of the LSF. In the model calculation, the contributions to line broadening due to the nonzero slit width, PSF broadening, and charge diffusion are included explicitly. Thus, the quantity $\sigma_{\mathrm{LSF}}$ in Equation (4) represents only the remaining portion of the point-source LSF, which is largely due to the roughness of the grating surface. This quantity cannot be measured directly but can be estimated by comparison with the linewidths measured from wavelength calibration lamp exposures.

The STIS Instrument Handbook (Leitherer et al. 2000) lists an expected extended-source LSF of FWHM $\approx 4$ pixels for the $0^{\prime \prime} 2$-wide slit. However, the comparison lamp exposures obtained with our data showed linewidths of FWHM $=3.3$ pixels. To estimate $\sigma_{\mathrm{LSF}}$, we computed a model for a monochromatic source of uniform surface brightness. The observed linewidths for the comparison lamp exposures were reproduced by setting $\sigma_{\mathrm{LSF}}$ in the range $5-8 \mathrm{~km} \mathrm{~s}^{-1}$. This represents a very small contribution to the total line broadening, so the model results are insensitive to the exact value of $\sigma_{\mathrm{LSF}}$.

Preliminary model runs (shown in Figure 11) indicated that the observed [N II] linewidths are too large to be merely the result of rotational, instrumental, and thermal broadening. In other

words, the gas in the disk must have a substantial bulk velocity dispersion, similar to what has been observed in circumnuclear disks in some other early-type galaxies (van der Marel \& van den Bosch 1998; Verdoes Kleijn et al. 2000). To match the run of linewidths over the face of the disk, 
we parameterize the radial component of this bulk velocity dispersion with the function

$$
\sigma_{r}(r)=\sigma_{0}+\sigma_{1} e^{-r / r_{0}}
$$

To find the optimal values for the three parameters $\sigma_{0}, \sigma_{1}$, and $r_{0}$, we calculated a set of models to minimize a goodness-of-fit parameter $\chi_{\sigma}^{2}$ that described the squared deviation between the model and observed linewidths. The best-fitting values are $\sigma_{0}=35 \mathrm{~km} \mathrm{~s}^{-1}, \sigma_{1}=105 \mathrm{~km} \mathrm{~s}^{-1}$, and $r_{0}=50$ pc. Since the intrinsic linewidth is the dominant source of line broadening in the data, these three parameters can be fixed at these values in the later model optimizations that we use to determine $M_{\bullet}$ and $\Upsilon$. In other words, models with different BH masses and hence different amounts of rotational broadening will still have essentially the same final linewidths because the intrinsic linewidth is significantly larger than the rotational broadening at most locations, and the different sources of broadening effectively add in quadrature.

The $\mathrm{H} \alpha$ velocity dispersion, on the other hand, is reproduced extremely well over most of the disk by a model for a cold disk with no bulk velocity dispersion (Figure 11). Only at the centers of slit positions 3 and 4 does the $\mathrm{H} \alpha$ velocity dispersion rise significantly above the cold disk model predictions. There is a great deal of scatter in the $\mathrm{H} \alpha$ linewidths at the center of slit position 3 , but the central region of slit position 4 can be fit reasonably well by a model having the same values of $\sigma_{1}$ and $r_{1}$ as in the [N II] model, but having $\sigma_{0}=0$. Differences in the widths of different emission lines have been observed in almost every ionized gas disk studied with HST data. In some cases it has been attributed to underlying differences in the spatial distribution of different ionic species, as a result of spatially unresolved density and/or ionization gradients (see discussion in Verdoes Kleijn et al. 2000). However, the STIS data shows that in NGC 3245, [N II] and $\mathrm{H} \alpha$ have different widths over the entire face of the disk. If this difference is not entirely due to the effects of underlying $\mathrm{H} \alpha$ absorption, then there must be spatially coincident but kinematically distinct components within the disk.

In the models for the dynamically cold disk (the dotted line in Figure 11), the line widths are determined only by the instrumental, rotational, and thermal broadening. At some positions in the model, the gradients in rotational velocity and in instrumental wavelength shifts across the slit are oppositely directed and partially cancel each other out, and the predicted line profiles become narrower than in surrounding areas. These regions are seen as the broad dips in velocity dispersion for the cold disk model, for example at $-0,5$ from the slit center in slit position 5 . In an extreme case, the instrumental and rotational broadening can cancel out almost exactly, yielding an extremely narrow linewidth in a localized region. Maciejewski \& Binney (2001) discuss these features in STIS spectra, which they refer to as "caustics," and they show that the position of a caustic can have diagnostic power for measuring the central mass. However, a substantial intrinsic velocity dispersion in the gas will blur out the caustic and make it difficult to detect in practice. Our model calculated for a dynamically cold disk predicts one caustic feature, at a location -0 '” 35 from the slit center in slit position 4. At this small distance from the nucleus, both [N II] and $\mathrm{H} \alpha$ have a large intrinsic velocity dispersion, and the caustic is not observed in either line. 


\subsection{Asymmetric Drift}

In common with some other galaxies having circumnuclear disks, NGC 3245 shares the trait that the intrinsic velocity dispersion of the emission-line gas rises dramatically toward the nucleus, at least for [N II]. This intrinsic linewidth is much larger than the thermal width for a cloud at $T \approx 10^{4} \mathrm{~K}$, so it may represent either local turbulence within the disk, or the combined motion of a system of collisionless clouds on eccentric orbits, similar to the stellar-dynamical case. It is important to address the possible dynamical effect of this velocity dispersion in the models. If it contributes a pressure which supports the disk against gravity, then the observed mean rotation speed $v_{\phi}(r)$ will deviate from the local circular velocity and models without an asymmetric drift correction will underestimate the true central mass.

The origin of the intrinsic velocity dispersion in nuclear gas disks is presently not understood, and it is not clear whether asymmetric drift plays a role in the dynamical structure of these objects. For IC 1459, where the nuclear disk becomes rounder at small radii and the innermost velocities appear to depart from a Keplerian curve, Verdoes Kleijn et al. (2000) suggest that asymmetric drift may be important. Similarly, Cretton, Rix, \& de Zeeuw (2000) describe an application of an asymmetric drift correction to the gas velocities in the elliptical galaxy NGC 2320, in order to derive the true circular velocity from the observed rotation velocity of the gas. On the other hand, most previous gas-dynamical $\mathrm{BH}$ measurements have assumed that the gas velocity dispersion results from a local "microturbulence," and that the gas still rotates at the circular velocity. Van der Marel \& van den Bosch (1998) argue that the gas velocity dispersion in NGC 7052 is due to local turbulence and does not affect the orbital structure. Without a clear understanding of the physical nature of the intrinsic velocity dispersion, we choose to calculate models both with and without an asymmetric drift correction, so that we can estimate the resultant uncertainty in the derived value of $M_{\bullet}$. The asymmetric drift is calculated using only the [N II] velocity dispersions, rather than $\mathrm{H} \alpha$, so as to estimate the maximum possible effect on the mass determination.

We assume that motions in the gas disk are close to isotropic in the $r$ and $z$ coordinates. Then, $\sigma_{z}=\sigma_{r}$ and $\left\langle v_{r} v_{z}\right\rangle=0$, and the asymmetric drift correction can be expressed as

$$
v_{c}^{2}-v_{\phi}^{2}=\sigma_{r}^{2}\left[-r \frac{d \ln \nu}{d r}-r \frac{d \ln \sigma_{r}^{2}}{d r}-\left(1-\frac{\sigma_{\phi}^{2}}{\sigma_{r}^{2}}\right)\right],
$$

where $\sigma_{\phi}$ is the azimuthal velocity dispersion, and $\nu(r)$ is the number density of gas clouds in the disk (e.g., Binney \& Tremaine 1987; Gilmore, King, \& van der Kruit 1990). Since the disk

surface brightness depends on proximity to the active nucleus and/or regions of star formation, the luminosity density in the disk is probably not a good tracer of the underlying number density of clouds. Nevertheless, for lack of a better alternative, we use the emission-line surface brightness distribution $S(r)$ to represent the cloud density.

In order to take the radial derivative of an analytic model rather than the noisy surface brightness profile itself, we use the exponential surface-brightness model to represent the number 
density of clouds in the disk. Using an analytic model of this kind is also preferable because the underlying number density of clouds in the disk is likely to be much more symmetric and more smoothly distributed than the patchy emission-line surface brightness. The relation between $\sigma_{r}$ and $\sigma_{\phi}$ is given by the epicycle approximation

$$
\frac{\sigma_{\phi}^{2}}{\sigma_{r}^{2}}=\frac{-B}{A-B},
$$

where $A$ and $B$ are the Oort constants (Binney \& Tremaine 1987). This approximation is strictly valid only in the limit $\sigma \ll v_{c}$, and for some other galaxies this method cannot be applied because $\sigma \approx v_{c}$ in the nuclear regions (e.g., IC 1459; Verdoes Kleijn et al. 2000). However, our best-fitting model for NGC 3245 has $\sigma_{r} / v_{c}<0.35$ everywhere in the disk (Figure 12), so this method can be adopted for at least an approximate treatment of the asymmetric drift. A more detailed assessment of the magnitude of the asymmetric drift would require dynamical modeling of the orbits of gas clouds in the disk, and such calculations are beyond the scope of this paper. In reality, the stellardynamical analogy must break down at some level because gas clouds in the disk will suffer collisions at a rate that depends on the typical cloud size and the filling factor of clouds in the disk. Thus, the actual mean rotation velocity in the disk will most likely be bracketed by the models with and without the asymmetric drift correction.

After calculating the asymmetric drift, we obtain the mean rotation velocity $v_{\phi}(r)$. The projected line-of-sight velocity field is calculated according to

$$
v_{p}=v_{\phi}\left(\frac{y^{\prime} \sin i}{r}\right)
$$

and the projected velocity dispersion along the line of sight is

$$
\sigma_{p}^{2}=\left(\sigma_{\phi}^{2}-\sigma_{r}^{2}\right)\left(\frac{y^{\prime} \sin i}{r}\right)^{2}+\sigma_{r}^{2}
$$

These values of $v_{p}$ and $\sigma_{p}$ are then used in the model calculation (equation 4). For models without an asymmetric drift correction, these relations reduce to $\sigma_{p}=\sigma_{\phi}=\sigma_{r}$ and $v_{\phi}=v_{c}$, corresponding to the case where the intrinsic velocity dispersion represents a locally isotropic microturbulence within the disk.

\subsection{Uncertainties and $\chi^{2}$ Calculation}

To optimize the models, we calculated a $\chi^{2}$ parameter for the model fit to the velocity curves. The linewidths and emission-line fluxes at each point carry little or no information about the possible presence of a $\mathrm{BH}$, so we do not include them in the $\chi^{2}$ calculation. The $\chi^{2}$ parameter was calculated over a rectangular region, and we define the "fitting radius" $r_{\text {fit }}$ as the maximum distance from the slit center over which $\chi^{2}$ was calculated for each slit. 
The initial uncertainties in the measured velocities are the values returned by the tripleGaussian fit to the $\mathrm{H} \alpha$ and [N II] emission lines. By forcing $\mathrm{H} \alpha$ and [N II] to have the same velocity in the fit, we effectively determine a best-fitting mean velocity for the blend, at the expense of underestimating the true uncertainty in the velocity measurements. In regions of high $\mathrm{S} / \mathrm{N}$, the formal model-fitting uncertainties are only a few $\mathrm{km} \mathrm{s}^{-1}$. At some locations, however, the velocities of $\mathrm{H} \alpha$ and $[\mathrm{N} \mathrm{II}]$ disagree by up to $\sim 30 \mathrm{~km} \mathrm{~s}^{-1}$. In addition, there are regions in the disk which systematically deviate from the circular rotation models for any reasonable model parameters. Since the formal error bars are much smaller than these local deviations from pure circular rotation, no model fit can be expected to yield $\chi^{2} / d \approx 1$. When deriving a goodness-of-fit parameter to optimize the model fits, we choose to rescale the error bars so as to incorporate these local deviations from pure circular rotation as effectively random errors in velocity. This "extra" uncertainty is independent of the $\mathrm{S} / \mathrm{N}$ of the data, as it depends only on the dynamical properties of the disk at a given location. Thus, we rescale the error bars by adding a single constant in quadrature to the velocity uncertainties. This constant was set to $10 \mathrm{~km} \mathrm{~s}^{-1}$ so that the best-fitting model with $r_{\text {fit }}$ $=0^{\prime \prime} .5$ would yield $\chi^{2} / d \approx 1.0$. Rescaling the error bars in this fashion is conservative in the sense that it increases the final uncertainty and widens the confidence intervals on $M_{\bullet}$.

\section{Velocity Field Modeling: Results}

To optimize the model fits, we performed $\chi^{2}$ minimizations using the downhill simplex algorithm described by Press et al. (1992). Initially, the five parameters $M_{\bullet}, \Upsilon, i, \theta$, and $v_{\mathrm{sys}}$ were allowed to vary independently in the minimization. We first discuss models without a correction for asymmetric drift. The preliminary models were calculated out to $r_{\text {fit }}=0$. 5 , giving a $1^{\prime \prime} \times 1^{\prime \prime} .2$ rectangular region. To test the effects of the grid sampling size, we ran models for values of $s$ from 1 to 6 . Different choices for $s$ lead to very similar results (Figure 13), with a root-mean-square (RMS) scatter in $M_{\bullet}$ of only $4 \%$ for $s$ in the range $1-6$. The models with $s=2$ yield the best fits, and we performed our final calculations using $s=2$. For $r_{\text {fit }}=0.5$ and $s=2$, there are 105 points in the velocity curves and the total $\chi^{2}$ of the best-fitting model is 102.3 .

After many trial runs with different initial parameter values, we found that the model fits consistently converged on $i=63^{\circ}$ and $\theta=27^{\circ}$. These values are consistent with expectations from the WFPC2 imaging data. To explore the range of uncertainty on the disk orientation parameters, we calculated a grid of models over a range of values of $i$ and $\theta$, leaving $M_{\bullet}, \Upsilon$, and $v_{\text {sys }}$ as free parameters in the fits. Figure 14 displays contours of constant $\chi^{2}$ for this model grid, demonstrating that with five parallel slit positions the gas kinematics can tightly constrain the disk orientation parameters (to better than $\pm 1^{\circ}$ at $68.3 \%$ confidence).

At radii beyond the edge of the disk, the ionized gas velocities begin to deviate systematically from circular motion. Beyond $\sim 150$ pc from the nucleus, the WFPC2 F547M image shows a filamentary structure in dust absorption which does not have obvious circular symmetry, and we do not attempt to fit kinematic models to this region. 
The galaxy's systemic velocity $v_{\text {sys }}$ was left as a free parameter and determined separately for each model calculation. From the best-fitting model, we find $v_{\mathrm{sys}}=1388 \mathrm{~km} \mathrm{~s}^{-1}$. Different model runs with reasonable parameters yielded results within a few $\mathrm{km} \mathrm{s}^{-1}$ of this value. Huchra et al. (1990) list a value of $1358 \pm 15 \mathrm{~km} \mathrm{~s}^{-1}$ for the recession velocity of NGC 3245. The discrepancy is most likely caused by the asymmetric distribution of emission-line surface brightness across the disk, which would bias a ground-based measurement toward the lower velocities of the northern side of the disk. For $v_{\text {sys }}=1388 \mathrm{~km} \mathrm{~s}^{-1}$, our model predicts a surface-brightness-weighted mean velocity of $1360 \mathrm{~km} \mathrm{~s}^{-1}$, in good agreement with the ground-based redshift.

In all of the calculations described above, the center of rotation was assumed to be at the same location as the peak of the $\mathrm{H} \alpha+[\mathrm{N} \mathrm{II}]$ surface brightness at the nucleus. As a test, we computed models in which the projected velocity field map was shifted in $x$ and $y$ relative to the emission-line surface brightness map. We found that shifting the velocity field by even one STIS pixel in any direction (i.e., $\sim 5 \mathrm{pc}$ ) led to significant increases in $\chi^{2}$, and we conclude that the dynamical center is effectively coincident with the optical peak in the WFPC2 $\mathrm{H} \alpha+[\mathrm{N} \mathrm{II}]$ image.

\section{1. $\quad$ Fitting Region and Best-Fitting Models}

The model fit with $s=2$ and $r_{\text {fit }}=00^{\prime \prime} 5$ gives a best-fit BH mass of $2.09 \times 10^{8} \mathrm{M}_{\odot}$ and $\Upsilon=3.74$ in $R$-band solar units. One way to gauge the uncertainty in the results is to run models with a range of values of $r_{\text {fit }}$. Fixing $i=63^{\circ}$ and $\theta=27^{\circ}$, we ran model fits with $r_{\text {fit }}$ ranging from $0^{\prime \prime} 2$ to $1^{\prime \prime} 0$. The results, shown in Figure 15, demonstrate that different choices of $r_{\text {fit }}$ can result in values of $M_{\bullet}$ in the range $(2.0-2.6) \times 10^{8} \mathrm{M}_{\odot}$, for models with no asymmetric drift. Figures 16 and 17 show the best-fitting models for $r_{\text {fit }}=0^{\prime \prime} .5$ and $r_{\text {fit }}=1^{\prime \prime} .0$, and also the best-fitting models for the case $M_{\bullet}=0$.

For large values of $r_{\text {fit }}$ (greater than about 0 "' 5 ), $\chi^{2}$ is dominated by points far from the $\mathrm{BH}$, and the models have relatively little sensitivity to $M_{\bullet}$. Also, with $r_{\text {fit }}>0$. 5 there are data points outside the optical edge of the disk, and these points appear to deviate systematically from any circular rotation model, particularly in slit position 5. The deviations from model predictions outside the main body of the disk are a possible sign of a warp, or the kinematic signature of gas which has not yet settled into the disk. Thus, the derived BH mass probably becomes unreliable as $r_{\text {fit }}$ increases beyond 0.5 .

Even in the inner portions of the disk, some points in the velocity curves deviate from the circular rotation models. For example, there is a "step" in the velocity curve for slit position 3 at $0{ }^{\prime \prime} 3$ south of the nucleus, and a region $00^{\prime \prime} 3-0 . \prime 6$ north of the nucleus has a rotation speed too large to be fit by the models. The influence of these regions on the model fits depends on the size of the fitting region, and partly explains the variation in the derived value of $M_{\bullet}$ for varying values of $r_{\text {fit }}$.

The dependence of $M_{\bullet}$ on $r_{\text {fit }}$ may also result at least in part from a real gradient in the $R$-band mass-to-light ratio across the inner arcsecond of the galaxy. As shown by Carollo et al. 
(1997), early-type galaxies often have radial variations in $V-I$ color of $0.1-0.2$ mag over the inner arcsecond. An intrinsic color gradient in NGC 3245 (presumably due to an underlying metallicity gradient) could conceivably lead to variations in $\Upsilon$ at the level observed in NGC 3245. Another possibility is that the inner disk may be slightly warped, so the mass derived from fitting planar disk models would depend on the fitting region.

We choose $r_{\text {fit }}=0$ ".5 for defining our "best-fit" value of $M_{\bullet}$, as this region is small enough to be entirely contained within the disk, but large enough to contain a sufficient number of data points that the model fits can be constrained tightly. In principle, one could attempt to select an "optimal" fitting region by finding the value of $r_{\text {fit }}$ that would lead to the minimum value of $\chi^{2} / d$. However, for this dataset we find that the minimum value of $\chi^{2} / d$ occurs at the smallest possible values of $r_{\text {fit }}$. As $r_{\text {fit }}$ increases, $\chi^{2} / d$ increases due to the influence of discrepant regions which depart slightly from circular rotation. By choosing $r_{\text {fit }}$ large enough to cover much of the disk, we ensure that any individual discrepant region will not have a substantial effect on the results. We note also that models fitted to only the three central slits result in values of $M$ • which differ by $\lesssim 5 \%$ from the values derived using all five slits.

Figure 18 shows the results of models calculated for $r_{\text {fit }}=0^{\prime \prime} 5$, for $M_{\bullet}$ ranging from 0 to $6 \times 10^{8}$ at intervals of $0.1 \times 10^{8} \mathrm{M}_{\odot}$. At each fixed value of $M_{\bullet}$, the parameters $\Upsilon, i, \theta$, and $v_{\text {sys }}$ were left as free parameters. For models without asymmetric drift, $M_{\bullet}$ is constrained to lie within $(1.8-2.3) \times 10^{8} \mathrm{M}_{\odot}$ at $68.3 \%$ confidence, and within $(1.5-2.7) \times 10^{8} \mathrm{M}_{\odot}$ at $99 \%$ confidence, with a best-fitting value of $2.1 \times 10^{8} \mathrm{M}_{\odot}$. Models without a central dark mass are ruled out at better than $99.99 \%$ confidence. With the asymmetric drift correction included, the best-fitting $\mathrm{BH}$ mass increases to $2.3 \times 10^{8} \mathrm{M}_{\odot}$, with $68.3 \%$ and $99 \%$ confidence ranges of $(2.1-2.5) \times 10^{8}$ and $(1.8-2.9) \times 10^{8} M_{\odot}$, respectively. Including the asymmetric drift correction increases the minimum value of $\chi^{2}$ from 102.3 to 106.9 . Since the models without asymmetric drift provide better fits, we use these models to derive the $\mathrm{BH}$ mass, but we allow for the possibility of asymmetric drift by including it in the estimated uncertainty range for $M_{\bullet}$.

The $\chi^{2}$ curve appears smooth despite the fact that the models are calcualted on a discrete spatial grid, because the values of $M_{\bullet}$ and $\Upsilon$ are continuous variables and $\chi^{2}$ can vary smoothly as these parameters change. We also note that the orientation parameters $i$ and $\theta$ are so tightly constrained that the curves of minimum $\chi^{2}$ in Figure 18 would appear nearly unchanged if these parameters were held fixed at their best-fitting values rather than being allowed to vary in the fits.

Over the range $r_{\text {fit }}=00^{\prime \prime} 2-00^{\prime \prime} 5$, the derived value of $\Upsilon$ is 3.5-3.8 in $R$-band solar units. This is comparable to $R$-band mass-to-light ratios observed in other early-type galaxies. For a sample of ellipticals, van der Marel (1991) found a mean value of $\Upsilon_{R}=(3.32 \pm 0.14) h_{50}$, where $h_{50}$ is the Hubble constant in units of $50 \mathrm{~km} \mathrm{~s}^{-1} \mathrm{Mpc}^{-1}$. 


\section{2. $\quad$ Error Budget}

We summarize the sources of uncertainty as follows.

Stellar profile fitting: We investigated the uncertainty in $M_{\bullet}$ due to the uncertainty in deprojecting the galaxy's surface brightness profile by running models in which the stellar circular velocity curve $v_{\star}$ was displaced by $\pm 1 \sigma$ from its default value. The confidence limits on $v_{\star}$ were determined by deprojecting different Monte Carlo realizations of the surface brightness profile, on the basis of the $1 \sigma$ uncertainty of each $R$-band isophote from the surface brightness profile fit. A total of 100 realizations of the surface brightness profile were used to derive the uncertainty on $v_{\star}$. For models with increased or decreased stellar surface brightness, the resulting change in $M_{\bullet}$ is (respectively) $-5.7 \%$ or $+1.9 \%$.

Model fitting uncertainty: The $68.3 \%$ confidence interval on $M_{\bullet}$ (corresponding to a $1 \sigma$ uncertainty) from the model fitting is $(1.8-2.3) \times 10^{8} \mathrm{M}_{\odot}$, for models without the asymmetric drift correction. This includes the (negligible) uncertainty due to the disk-orientation parameters $i$ and $\theta$, which were allowed to vary in the model fits.

Asymmetric Drift: The asymmetric drift correction raises the best-fitting BH mass by $0.25 \times 10^{8}$ $\mathrm{M}_{\odot}$, or $12 \%$. However, it is not clear whether there actually is asymmetric drift in the NGC 3245 disk. To account for the possibility that there may be asymmetric drift, we propagate a $12 \%$ uncertainty into the upper error bar on $M_{\bullet}$.

Distance: The mass derived from modeling the velocity field is proportional to $D$. Tonry et al. (2001) give an uncertainty of $0.20 \mathrm{mag}$ in the distance modulus to NGC 3245, corresponding to a $10 \%$ uncertainty in distance. In addition, there is a systematic uncertainty of $9 \%$ in the Cepheidbased zeropoint of the distance scale (Mould et al. 2000). We propagate only the random error in the distance into our result, since changes in the zeropoint of the distance scale will affect all $\mathrm{BH}$ mass measurements equally.

Fitting radius: A major source of uncertainty in $M_{\bullet}$ is the choice of fitting radius. For $r_{\text {fit }}$ in the range $0^{\prime \prime} 2-0^{\prime \prime} 5, M_{\bullet}$ varies between $2.0 \times 10^{8}$ and $2.3 \times 10^{8} \mathrm{M}_{\odot}$, and we propagate this range into the $1 \sigma$ uncertainty on $M_{\bullet}$. As $r_{\text {fit }}$ increases beyond $0^{\prime \prime} 5, M_{\bullet}$ rises to $\geq 2.4 \times 10^{8} \mathrm{M}_{\odot}$, but for such large fitting regions the results are unreliable because of noncircular motions outside the disk, and because the fit is dominated by points far from the BH.

Subsampling factor: For $r_{\text {fit }}=0$ ". 5 , models computed with different values of $s$ have an RMS scatter of $9 \times 10^{6} \mathrm{M}_{\odot}$, or $4 \%$ of the best-fitting BH mass. This variation results from the graininess of the calculation and the differences in the $\mathrm{H} \alpha+[\mathrm{N}$ II] surface-brightness model for different subsampling factors. We propagate this RMS scatter into our final uncertainty range.

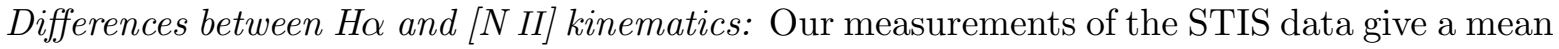
velocity from the $\mathrm{H} \alpha$ and [N II] emission lines together, and we have not attempted to measure or fit models to the individual velocity curves of $\mathrm{H} \alpha$ and $[\mathrm{N} \mathrm{II}]$. As discussed in $\S 4.5$, these two lines 
have different widths, and the models described above were calculated using the intrinsic velocity dispersion that provided the best fit to the $[\mathrm{N} \mathrm{II}]$ data. We also ran a model in which the intrinsic velocity dispersion of the gas was set to match $\mathrm{H} \alpha$ rather than [N II], to test whether this would change the result substantially. The model fit converged on $M_{\bullet}=1.91 \times 10^{8} \mathrm{M}_{\odot}$, or $9 \%$ lower than the best-fitting mass for our standard models. To account for the possibility of differences in the kinematics of different ionic species, we propagate an uncertainty of $9 \%$ into our final result.

Combining all of these sources of uncertainty, we arrive at a final range of $M_{\bullet}=(1.6-2.6) \times 10^{8}$ $\mathrm{M}_{\odot}$, with a best-fit value of $2.1 \times 10^{8} \mathrm{M}_{\odot}$. This mass range is meant to correspond to a $1 \sigma$ confidence limit, although the uncertainty range due to the choice of fitting radius is somewhat subjective and cannot be expressed in terms of formal confidence intervals.

One additional potential source of uncertainty is the correction for dust extinction in the $R$ band image. Had we measured the stellar profile without correcting for extinction at all, the derived $\mathrm{BH}$ mass would have been $2.6 \times 10^{8} \mathrm{M}_{\odot}$. While we do not attempt to quantify the uncertainty in the extinction correction, the fact that the entire correction only changes the BH mass by less than $25 \%$ suggests that the uncertainty in the correction will not be a significant contributor to the overall error budget.

\subsection{How Compact is the Central Dark Mass?}

Even if a central dark mass is detected, our observations cannot determine whether it is truly a single compact object. To test the possible spatial extent of the dark mass, we computed models using a Plummer model to represent the density distribution of the dark mass (e.g., Macchetto et al. 1997; Maoz 1998). This model is an extreme case in the sense of having a very low central density for a given half-mass radius. For a given central mass, the rotation curves for these extended-mass models are identical at large radii to the models with a point-like central mass, but at small radii the central slopes are shallower. We ran model fits for values of the half-mass radius $\left(r_{1 / 2}\right)$ of the Plummer model ranging from 0 to $20 \mathrm{pc}$ at intervals of $1 \mathrm{pc}$ (Figure 19). The models were run with $s=4$ to improve the spatial sampling at the nucleus.

The best-fitting models are obtained for a pointlike central mass. As $r_{1 / 2}$ increases beyond 1 pc, the total dark mass required to fit the central velocity slope rises correspondingly. The increase in $\chi^{2}$ indicates that the central mass must have $r_{1 / 2}<7 \mathrm{pc}$ at $68.3 \%$ confidence, or $r_{1 / 2}<12 \mathrm{pc}$ at $99 \%$ confidence. The corresponding density of the dark mass must be $>1.0 \times 10^{5} \mathrm{M}_{\odot} \mathrm{pc}^{-3}$ at $68.3 \%$ confidence, or $>2.3 \times 10^{4} \mathrm{M}_{\odot} \mathrm{pc}^{-3}$ at $99 \%$ confidence, within the half-mass radius. This is comparable to the central densities found in most other BH hosts, but significantly lower than the inferred central densities for galaxies such as M87 in which the BH sphere of influence is better resolved (see Maoz 1998). 


\subsection{Comparison of Models with Different Features}

To determine whether the level of detail in our modeling code actually provides a noticeable improvement in the final results, we also ran a series of models where various features of the code were "turned off." We calculated models in which the $\mathrm{H} \alpha+[\mathrm{N}$ II] surface brightness was set to a constant, or set to the exponential surface-brightness model; models in which the PSF was set to a delta function at the limit of the model resolution; models in which the instrumental wavelength shifts of light entering the slit off-center were omitted; and models in which the STIS pixels were not subsampled. In all of these calculations, we used $r_{\text {fit }}=0.5$.

As shown in Table 2, the instrumental wavelength shifts alone do not result in a substantial change in the derived BH mass, but including them does improve the fits to the observed rotation curves. Neglecting the PSF structure can lead to dramatically different model results, however. In general, models which do not include blurring effects (the PSF and nonzero aperture size) will tend to underestimate the $\mathrm{BH}$ mass because a properly blurred model will require a greater central mass to match a given rotation velocity.

The model results are also sensitive to the treatment of the emission-line surface brightness. In particular, use of the exponential surface-brightness model led to difficulties. For $s=1$, the model velocity curves appear to fit the data adequately (Figure 20), but at higher values of $s$, the increased peakiness of the central surface brightness led to large velocity deviations in the innermost regions of slit position 3. For high values of the subsampling factor, reasonable results could only be obtained if the exponential surface-brightness profile was assumed to flatten out in the inner core, or if there was an inner "hole" in the $\mathrm{H} \alpha+[\mathrm{N} \mathrm{II}]$ distribution. This problem is not unique to the exponential model; it would occur for any other model with a comparably steep profile at the nucleus. The $\mathrm{H} \alpha+[\mathrm{N} \mathrm{II}]$ surface brightness model derived from the WFPC2 image does not suffer from this problem because its nuclear profile is not nearly as sharply peaked. With the exponential surface-brightness model and $s=1$, the best-fitting BH mass is $1.5 \times 10^{8} \mathrm{M}_{\odot}$, significantly below the best-fitting result for models incorporating the actual $\mathrm{H} \alpha+[\mathrm{N}$ II] surface-brightness distribution. The radial velocity curves in Figure 10 also illustrate the importance of including an accurate model for the surface-brightness distribution.

Notably, the lowest value of $\chi^{2}$ was found for the "full" models which included instrumental wavelength shifts, the Tiny Tim PSF, and the actual H $\alpha+[\mathrm{N}$ II] surface brightness derived from the WFPC2 F658N image. None of these features adds any free parameters to the fit, so the decrease in $\chi^{2}$ represents a real and significant improvement in model results when all available information is taken into account in the modeling code. In comparison with our best-fitting model, all models using the exponential surface-brightness profile are excluded at $>99.99 \%$ confidence.

These results underscore the need for accurate modeling in future gas-dynamical BH measurements. The narrow-band image can be a particularly important input to the models, especially for galaxies such as NGC 3245 in which the emission-line surface brightness is patchy or irregular. Using the 0 '! 1 -wide slit would alleviate these problems to some extent, but at the expense of reduced 
$\mathrm{S} / \mathrm{N}$. Also, we note that many of the features in our modeling procedure should be equally applicable to stellar-dynamical BH measurements. Instrumental wavelength shifts and separation of the PSF into optical and charge-diffusion components will affect the line-of-sight velocity profiles and velocity dispersions derived from stellar absorption lines, but stellar-dynamical BH measurements done with HST data have generally not treated instrumental effects at this level of detail.

\section{Discussion}

\subsection{Is the Central Dark Mass a BH?}

With numerous recent claims of detections of BHs in galactic nuclei based on HST data, it is important to remember that these observations cannot prove that a $\mathrm{BH}$ has actually been detected. Strictly speaking, we have detected a massive dark object and determined a lower limit to its density. At best, our data probe scales of $\sim 10^{5}$ Schwarzschild radii, and at this resolution we cannot definitively rule out alternatives to the BH interpretation. Maoz (1998) has shown that a hypothetical cluster of dark objects such as stellar-mass BHs, neutron stars, brown dwarfs, or planetary-mass objects could in principle have an extremely long lifetime against evaporation or collisions. The nuclei of our Galaxy and NGC 4258 are the only two cases where the inferred central densities are sufficiently great that any hypothetical dark cluster would have a lifetime shorter than the Hubble time. While such "dark cluster" models may not seem plausible or well motivated, they are also not ruled out by the available data. For most claimed BH detections including NGC 3245, the conclusion that the massive dark object is a $\mathrm{BH}$ still rests on circumstantial evidence and on the expectation that a supermassive $\mathrm{BH}$ ought to be present in the nuclei of most massive galaxies (e.g., Soltan 1982).

\subsection{Asymmetric Drift and the Intrinsic Velocity Dispersion}

The origin of the intrinsic velocity dispersion in nuclear gas disks remains a puzzling issue, particularly in light of the differing velocity dispersions of $\mathrm{H} \alpha$ and [N II] in NGC 3245. The fact that the $[\mathrm{N} \mathrm{II}] / \mathrm{H} \alpha$ intensity ratio is highest in regions having the highest velocity dispersion suggests that shock excitation may be responsible for the LINER emission near the center of the disk. This possibility could be tested with observations of a wider range of emission lines. It is reassuring that the asymmetric drift correction does not lead to a very substantial change in $M$. for NGC 3245 when calculated for [N II]; for $\mathrm{H} \alpha$ the inferred asymmetric drift would be an even smaller effect. However, the effect may be more important in other galaxies. In IC 1459 the gas velocity dispersion rises to $\sim 500 \mathrm{~km} \mathrm{~s}^{-1}$ at the nucleus, and if there is asymmetric drift then the BH mass could be a factor of $\sim 3-4$ greater than the mass derived from cold disk models (Verdoes Kleijn et al. 2000). This does make the BH detection more secure, but raises the uncertainty on $M_{\bullet}$ far beyond the level of the uncertainty in the model fitting. The nuclear disk in M87, on the other 
hand, does not appear to have a substantial intrinsic velocity dispersion relative to the circular velocity (Macchetto et al. 1997).

It appears that asymmetric drift may play an important role in the dynamical structure of some, but perhaps not all, nuclear gas disks. With new results from the various STIS programs currently in progress, it should be possible to determine whether there is any relation between host galaxy properties and the intrinsic velocity dispersion in nuclear disks. For example, is the ratio $\sigma / v_{c}$ in nuclear disks correlated with $M_{\bullet}$ or with bulge properties? Also, can any galaxies be found in which an asymmetric drift correction improves the model fits? If asymmetric drift does turn out to be an important effect in many nuclear disks, then some previous BH mass measurements might have underestimated the true $\mathrm{BH}$ masses by a substantial factor.

\subsection{Host Galaxy Properties}

Gebhardt et al. (2000) and Ferrarese \& Merritt (2000) (see also Merritt \& Ferrarese 2001) have shown that there is a very small scatter in the relation between $M_{\bullet}$ and bulge velocity dispersion, at least for the galaxy samples they studied. Having additional BH mass measurements makes it possible to refine and test these correlations. For NGC 3245, the central velocity dispersion $\left(\sigma_{c}\right)$ is $230 \mathrm{~km} \mathrm{~s}^{-1}$ (Tonry \& Davis 1981). The relation derived by Merritt \& Ferrarese (2001) predicts $M_{\bullet}=2.5 \times 10^{8} \mathrm{M}_{\odot}$ for this velocity dispersion. The $M_{\bullet}-\sigma$ relation derived by Gebhardt et al. (2000) uses the surface-brightness weighted velocity dispersion within the effective radius $\left(\sigma_{e}\right)$, and we do not have a measurement of this quantity. However, as Gebhardt et al. (2000) show, the difference between $\sigma_{e}$ and $\sigma_{c}$ is typically smaller than $10 \%$. Assuming that $\sigma_{e}$ is close to $230 \mathrm{~km}$

$\mathrm{s}^{-1}$, the Gebhardt et al. (2000) relation predicts $M_{\bullet}=2.0 \times 10^{8} M_{\odot}$, with an uncertainty of $\sim 30 \%$ due to the possible difference between $\sigma_{e}$ and $\sigma_{c}$. Our results further support the predictive power of the $M_{\bullet}-\sigma$ relation, as both the Gebhardt et al. and the Merritt \& Ferrarese relations predict values of $M_{\bullet}$ which are within the $1 \sigma$ uncertainty range for NGC 3245.

NGC 3245 has an extinction-corrected absolute magnitude of $M_{B}=-20.08$ (Ho, Filippenko, \& Sargent 1997a), which corresponds to a $B$-band luminosity of $1.5 \times 10^{10} L_{\odot}$. The bulge accounts for $76 \%$ of this total $B$-band luminosity, as determined by using the bulge-disk decomposition parameters from Baggett, Baggett, \& Anderson (1998) to subtract off the disk contribution. Assuming a typical $B$-band mass-to-light ratio of 6 (van der Marel 1991), the bulge mass is $\sim 6.8 \times 10^{10} \mathrm{M}_{\odot}$. Then, $M_{\bullet} / M_{\text {bulge }} \approx 0.003$. The median $M_{\bullet} / M_{\text {bulge }}$ seen in other galaxies is $\sim 0.002$, but there is nearly an order of magnitude of scatter about this best-fit ratio (Ho 1999).

\section{Conclusions}

We have detected a massive dark object, presumably a BH, in the nucleus of NGC 3245. Its mass is constrained to be in the range $(1.6-2.6) \times 10^{8} \mathrm{M}_{\odot}$, and the $R$-band mass-to-light ratio 
is in the range $3.5-3.8$ in solar units. Models without a central dark mass predict radial velocity curves which are too shallow to match the data in the innermost regions, and are excluded at a formal confidence level better than $99.99 \%$. The STIS data provide excellent spatial coverage of the nuclear disk and high $\mathrm{S} / \mathrm{N}$, so we are able to verify that the disk kinematics are well matched by circular rotation models.

Our results demonstrate the importance of modeling the effects of the telescope and spectrograph optics, so that dynamical models for the rotating gas disk can be adequately compared with the data. Similar issues have also been discussed by van der Marel \& van den Bosch (1998) and Maciejewski \& Binney (2001). Models which do not take into account the nonzero aperture size or the PSF may be prone to unpredictable errors. Modeling the apparent wavelength shifts for light entering the slit off-center does not lead to significant changes in the derived value of $M_{\bullet}$, but it does improve the quality of the model fits, and allows the models to make detailed predictions for the line widths. A new aspect of our modeling technique is the use of the measured surface brightness distribution of emission-line light in the calculations, rather than a smooth approximation. Folding the WFPC2 narrow-band image into the models improves the quality of the fits significantly, and demonstrates that some of the apparent irregularities in the velocity field are actually due to local variations in surface brightness rather than local departures from circular rotation. We have also included other minor effects such as anamorphic magnification in the spectrograph optics and the separation of the PSF into optical and charge-bleeding components. While these do not alter the results dramatically, they are real effects which do occur in the spectrograph and they can easily be modeled without increasing the computation time.

For future progress in gas-dynamical BH mass measurements, careful choice of targets is crucial, because fewer than $20 \%$ of disk galaxies have emission-line velocity fields which are amenable to this type of analysis (Sarzi et al. 2001). On the theoretical front, the question of the dynamical state of these nuclear disks and the possible role of asymmetric drift remain important unresolved issues. Also, variations in the kinematics of different emission lines should be investigated further. Despite these difficulties, our results demonstrate that the gas-dynamical method can be used to measure BH masses to better than $25 \%$ accuracy with HST STIS data. We plan to apply these techniques to a larger sample of galaxies in the future, as a step toward filling in the $M_{\bullet}-\sigma$ correlation over the entire mass range of BHs in galactic nuclei.

Support for this work was provided by NASA through grant number GO-07403 from STScI, which is operated by AURA, Inc., under NASA contract NAS 5-26555. NASA grant NAG5-3556 is also acknowledged. Research by A.J.B. is supported by a postdoctoral fellowship from the HarvardSmithsonian Center for Astrophysics. We thank Walter Dehnen, Krzysztof Stanek, and Roeland van der Marel for illuminating discussions, and John Tonry for providing the SBF distance to NGC 3245 prior to publication. A.J.B. is grateful to the Max-Planck-Institut für Astronomie for its hospitality during a visit in September 2000. We thank the referee, Gary Bower, for several helpful suggestions which improved the presentation of this paper. 


\section{REFERENCES}

Adams, F. C., Graff, D. S., \& Richstone, D. O. 2001, ApJ, submitted

Baggett, W. E., Baggett, S. M., \& Anderson, K. S. J. 1998, AJ, 116, 1626

Bertola, F., Cappellari, M., Funes, J. G., Corsini, E. M., Pizzella, A., \& Vega Beltran, J. C. 1998, ApJ, 509, L93

Binney, J., \& Tremaine, S. 1987, Galactic Dynamics (Princeton: Princeton University Press)

Bower, G. A., et al. 1998, ApJ, 492, L111

Bowers, C., \& Baum, S. 1998, STIS Instrument Science Report 98-23 (Baltimore: STScI)

Cardelli, J. A., Clayton, G. C., \& Mathis, J. S. 1989, ApJ, 345, 245

Carollo, C. M., Franx, M., Illingworth, G. D., \& Forbes, D. A. 1997, ApJ, 481, 710

Corsini, E. M., Pizzella, A., Sarzi, M., Cinzano, P., Vega Beltrán, J. C., Funes, J. G., Bertola, F., Persic, M., \& Salucci, P. 1999, å, 342, 671

Cretton, N., Rix, H.-W., \& de Zeeuw, P. T. 2000, ApJ, 536, 319

Dopita, M. A., Koratkar, A. P., Allen, M. G., Tsvetanov, Z. I., Ford, H. C., Bicknell, G. V., \& Sutherland, R. S. 1997, ApJ, 490, 202

Emsellem, E., Monnet, G., Bacon, R., \& Nieto, J.-L. 1994, A\&A, 285, 739

Ferrarese, L., \& Ford, H. C. 1999, ApJ, 515, 583

Ferrarese, L., Ford, H. C., \& Jaffe, W. 1996, ApJ, 470, 444

Ferrarese, L., \& Merritt, D. 2000, ApJ, 539, L9

Gebhardt, K., et al. 2000, ApJ, 539, L13

Gilmore, G., King, I. R., \& van der Kruit, P. C. 1990, The Milky Way as a Galaxy (Mill Valley: University Science Books)

Goudfrooij, P., \& de Jong, T. 1995, A\&A, 298, 784

Haehnelt, M. G., \& Kauffmann, G. 2001, MNRAS, submitted

Harms, R. J., et al. 1994, ApJ, 435, L35

Ho, L. C. 1999, in Observational Evidence for Black Holes in the Universe, ed. S. K. Chakrabarti (Dordrecht: Kluwer), 157

Ho, L. C., Filippenko, A. V., \& Sargent, W. L. W. 1993, ApJ, 417, 63 
Ho, L. C., Filippenko, A. V., \& Sargent, W. L. W. 1995, ApJS, 98, 477

Ho, L. C., Filippenko, A. V., \& Sargent, W. L. W. 1997a, ApJS, 112, 315

Ho, L. C., Filippenko, A. V., Sargent, W. L. W., \& Peng, C. Y. 1997b, ApJS, 112, 391

Holtzman, J. A., Burrows, C. J., Casertano, S., Hester, J. J., Trauger, J. T., Watson, A. M., \& Worthey, G. 1995, PASP, 107, 1065

Huchra, J. P., Geller, M. J., de Lapparent, V., \& Corwin, H. G. 1990, ApJS, 72, 433

Jaffe, W., Ford, H. C., Ferrarese, L., van den Bosch, F., \& O’Connell, R. W. 1993, Nature, 364, 213

Kormendy, J. 2001, in Galaxy Disks and Disk Galaxies, ed. J. G. Funes \& E. M. Corsini (San Francisco: ASP), in press

Kormendy, J., \& Richstone, D. 1995, ARA\&A, 33, 581

Krist, J., \& Hook, R. 1999, The Tiny Tim User's Guide (Baltimore: STScI)

Leitherer, C., et al. 2000, STIS Instrument Handbook Version 4.0 (Baltimore: STScI)

Macchetto, F., Marconi, A., Axon, D. J., Capetti, A., Sparks, W., \& Crane, P. 1997, ApJ, 489, 579

Maciejewski, W., \& Binney, J. 2001, MNRAS, submitted

Maoz, E. 1998, ApJ, 494, L181

Martel, A. R., Turner, N. J., Sparks, W. B., \& Baum, S. A. 2000, ApJS, 130, 267

Merritt, D., \& Ferrarese, L. 2001, ApJ, in press

Michard, R., \& Poulain, P. 2000, A\&AS, 141, 1

Monnet, G., Bacon, R., \& Emsellem, E. 1992, A\&A, 253, 366

Mould, J. R., et al. 2000, ApJ, 529, 786

Osterbrock, D. E. 1989, Astrophysics of Gaseous Nebulae and Active Galactic Nuclei (Mill Valley: University Science Books)

Ostriker, J. P. 2000, Phys. Rev. Lett., 84, 5258

Press, W. H., Teukolsky, S. A., Vetterling, W. T., \& Flannery, B. P. 1992, Numerical Recipes (Cambridge: Cambridge University Press)

Richstone, D., et al. 1998, Nature, 395, A14

Sadler, E. M., \& Gerhard, O. E. 1985, MNRAS, 214, 177 
Sarzi, M., Rix, H.-W., Shields, J. C., Rudnick, G., Ho, L. C., McIntosh, D., Filippenko, A. V., \& Sargent, W. L. W. 2001, ApJ, in press

Schlegel, D. J., Finkbeiner, D. P., \& Davis, M. 1998, ApJ, 500, 525

Shields, J. C., Rix, H.-W., McIntosh, D. H., Ho, L. C., Rudnick, G., Filippenko, A. V., Sargent, W. L. W., \& Sarzi, M. 2000, ApJ, 534, L27

Silk, J., \& Rees, M. J. 1998, A\&A, 331, L1

Simien, F., \& Prugniel, P. 1998, A\&AS, 131, 287

Sołtan, A. 1982, MNRAS, 200, 115

Tonry, J. L., \& Davis, M. 1981, ApJ, 246, 666

Tonry, J. L., Dressler, A., Blakeslee, J. P., Ajhar, E. A., Fletcher, A. B., Luppino, G. A., Metzger, M. R., \& Moore, C. B. 2001, ApJ, 546, 681

van der Marel, R. P. 1991, MNRAS, 253, 710

van der Marel, R. P., \& van den Bosch, F. C. 1998, AJ, 116, 2220

Verdoes Kleijn, G. A., van der Marel, R. P., Carollo, C. M., \& de Zeeuw, P. T. 2000, AJ, 120, 1221

Wrobel, J. M., \& Heeschen, D. S. 1991, AJ, 101, 148

Young, J. S., Xie, S., Kenney, J. D. P., \& Rice, W. L. 1989, ApJS, 70, 699 
Table 1. Summary of STIS Observations

\begin{tabular}{lcrc}
\hline \hline $\begin{array}{c}\text { Slit } \\
\text { Position }\end{array}$ & $\begin{array}{c}\text { HST Image } \\
\text { Rootname }\end{array}$ & $\begin{array}{c}\text { Offset } \\
(\operatorname{arcsec})\end{array}$ & $\begin{array}{c}\text { Exposure Time } \\
(\mathrm{s})\end{array}$ \\
\hline 1 & 057205010 & 0.50 & 2961 \\
2 & 057205020 & 0.25 & 2715 \\
3 & $\mathrm{o} 57205030$ & 0.00 & 2715 \\
4 & $\mathrm{o} 57205040$ & -0.25 & 2715 \\
5 & $\mathrm{o} 57205050$ & -0.50 & 4651 \\
\hline
\end{tabular}

Note. - The listed offsets give the position of the slit center relative to the location of the target peakup. The offsets are oriented along P.A. $=112^{\circ}$, and positive offsets correspond to slit positions located east of the nucleus, as shown in Figure 1. 
Table 2. Comparison of Models with Different Features

\begin{tabular}{|c|c|c|c|c|c|c|c|c|}
\hline $\begin{array}{c}\text { Surface } \\
\text { Brightness }\end{array}$ & PSF & $\begin{array}{c}\text { Wavelength } \\
\text { Shifts? }\end{array}$ & $\begin{array}{c}\text { Subsampling } \\
\text { Factor }\end{array}$ & $\begin{array}{c}M_{\bullet} \\
\left(\mathrm{M}_{\odot}\right)\end{array}$ & $\begin{array}{c}\Upsilon \\
\left(\Upsilon_{\odot}\right)\end{array}$ & $\begin{array}{c}i \\
\text { (degrees) }\end{array}$ & $\begin{array}{c}\theta \\
(\text { degrees) }\end{array}$ & $\chi^{2}$ \\
\hline Constant & $\delta(r)$ & No & 2 & $1.18 \times 10^{8}$ & 4.03 & 63 & 27 & 143.6 \\
\hline Exponential & $\delta(r)$ & No & 2 & 0.0 & 4.55 & 63 & 26 & 181.8 \\
\hline Exponential & TinyTim & No & 1 & $1.32 \times 10^{8}$ & 4.21 & 62 & 26 & 142.2 \\
\hline Exponential & TinyTim & Yes & 1 & $1.49 \times 10^{8}$ & 4.10 & 63 & 26 & 145.7 \\
\hline Exponential & TinyTim & Yes & 2 & $8.12 \times 10^{7}$ & 4.34 & 64 & 26 & 181.3 \\
\hline Exponential & TinyTim & Yes & 4 & $4.89 \times 10^{7}$ & 4.61 & 65 & 26 & 207.0 \\
\hline WFPC2 & TinyTim & No & 2 & $2.04 \times 10^{8}$ & 3.79 & 63 & 27 & 115.9 \\
\hline WFPC2 & TinyTim & Yes & 2 & $2.09 \times 10^{8}$ & 3.74 & 63 & 27 & 102.3 \\
\hline
\end{tabular}

Note. - All models were calculated for $r_{\text {fit }}=0.5$, with no asymmetric drift correction, and with the four parameters $M_{\bullet}, \Upsilon, i$, and $\theta$ allowed to float in the model fits. For the $\mathrm{H} \alpha+[\mathrm{N}$ II] surface brightness, "Exponential" refers to a parameterization of the form $S_{0}+S_{1} e^{-r / r_{1}}$, and "WFPC2" refers to the surface brightness taken from the deconvolved, continuum-subtracted WFPC2 F658N image. The PSF was taken to be either a delta function at the limit of the model resolution, or a STIS PSF generated by the Tiny Tim program. The entry "Wavelength Shifts" indicates whether the models include instrumental shifts in wavelength for light entering the slit off-center. The last entry in the table is for a model with the real $\mathrm{H} \alpha+[\mathrm{N}$ II] profile, the Tiny Tim PSF, and instrumental wavelength shifts included; note that it results in the lowest value of $\chi^{2}$ by a significant margin. 


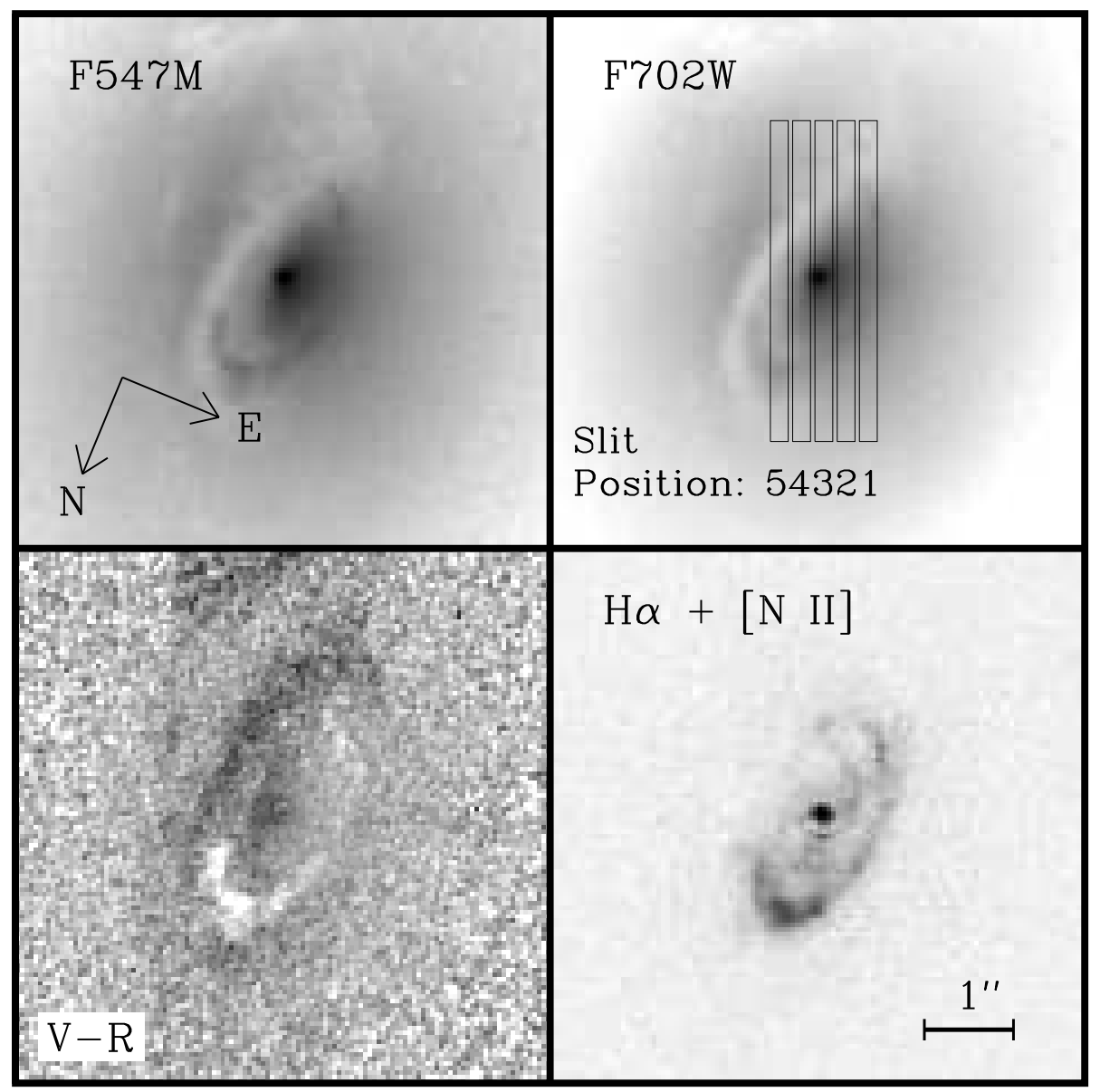

Fig. 1.- HST WFPC2/PC images of the nuclear region of NGC 3245. The images have been rotated to the STIS instrumental frame. The rectangles overplotted on the F702W images show the slit positions used in the spectroscopic exposures. Slit position 1 is the easternmost position, and each successive spectroscopic observation moved the slit aperture 0.25 to the northwest. The STIS instrumental $y$ axis points upward in this figure. In the $V-R$ image, dark regions are redder, and light regions are bluer than the surrounding starlight. The darkest regions correspond to a reddening of $E(V-R) \approx 0.3$ mag relative to the unreddened regions. Each box is 5".4 on a side. 


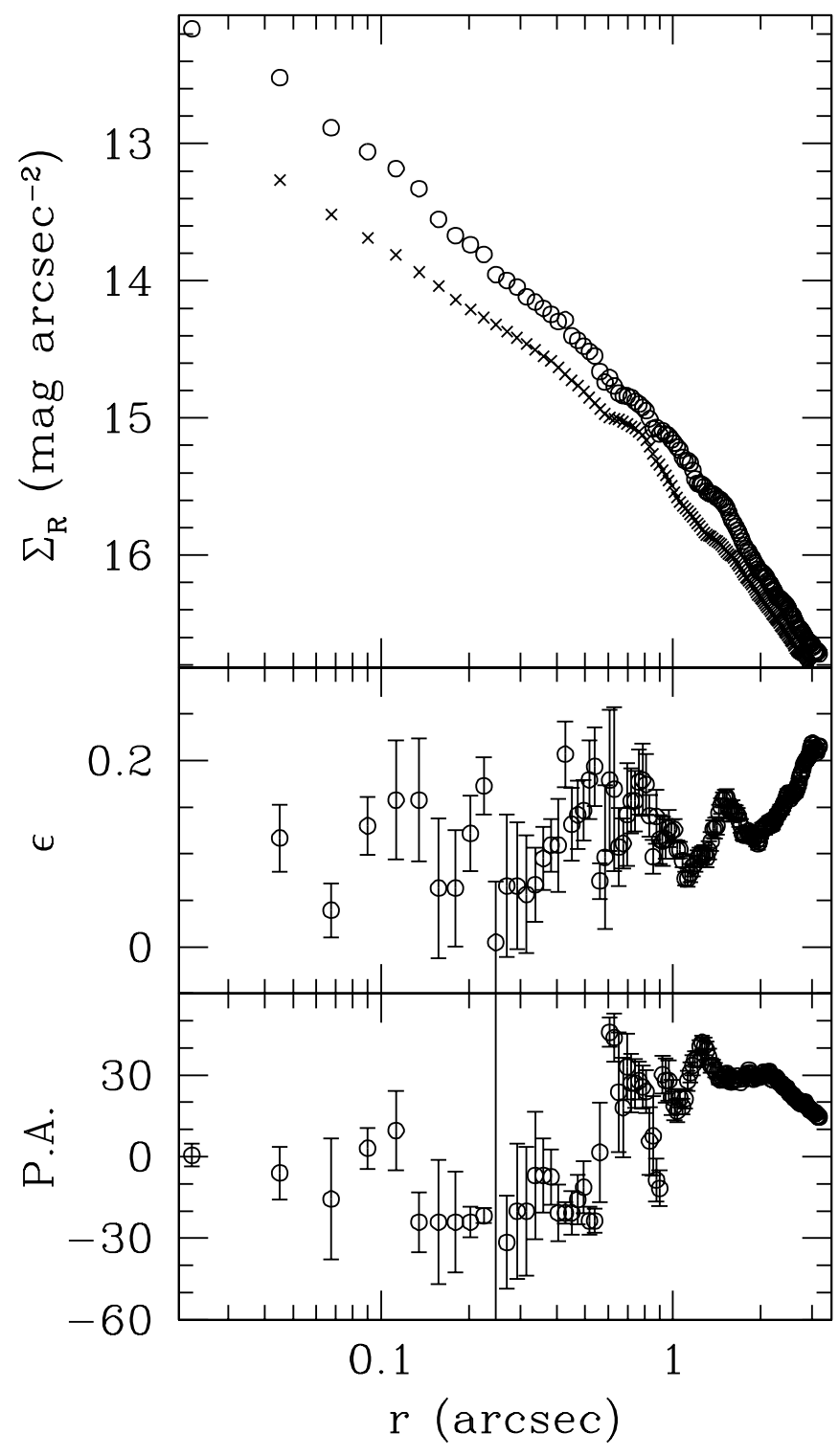

Fig. 2. - Radial profiles of $R$-band surface brightness, ellipticity, and position angle in NGC 3245, from the extinction-corrected WFPC2 F702W image. The position angle is given in degrees east from north. In the top panel, crosses represent the surface brightness prior to the extinction correction. Measurement uncertainties from isophote fitting are smaller than the plot symbols in the top panel. 


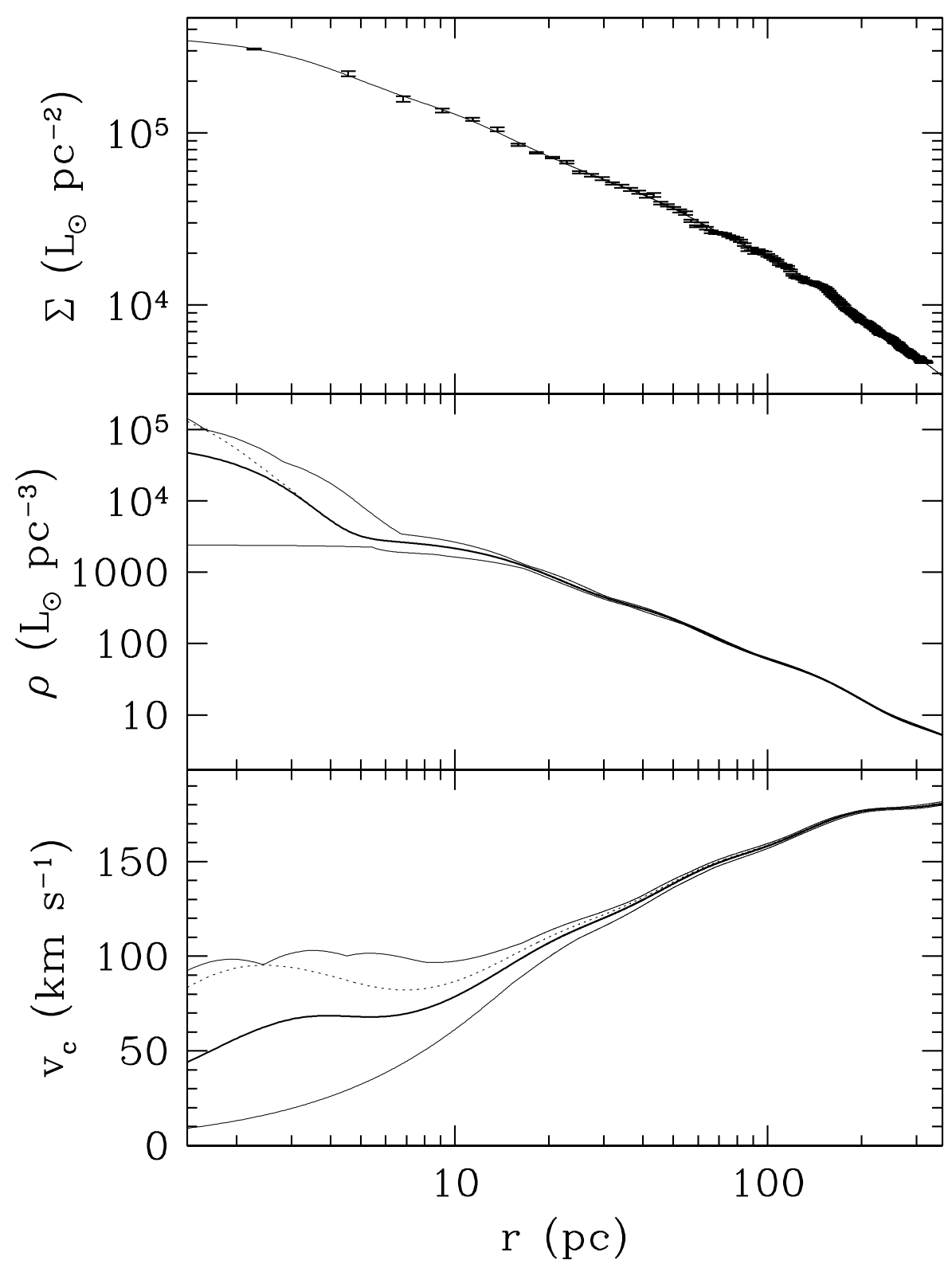

Fig. 3.- Deprojection steps for the stellar mass profile of NGC 3245. Top panel: Multi-Gaussian fit to the observed surface-brightness distribution $\Sigma(r)$. Middle panel: Deprojected intrinsic stellar luminosity profile $\rho(r)$ (thick line), and $3 \sigma$ confidence limits on $\rho$ (thin lines). Bottom panel: Circular velocity profile $v_{c}(r)$ obtained from $\rho(r)$ assuming $\Upsilon=1$ in $R$-band solar units, along with $3 \sigma$ confidence limits. In the middle and bottom panels, the dotted line shows the contribution of the nuclear point source, if it is assumed to be a stellar cluster rather than nonstellar emission from the active nucleus. 


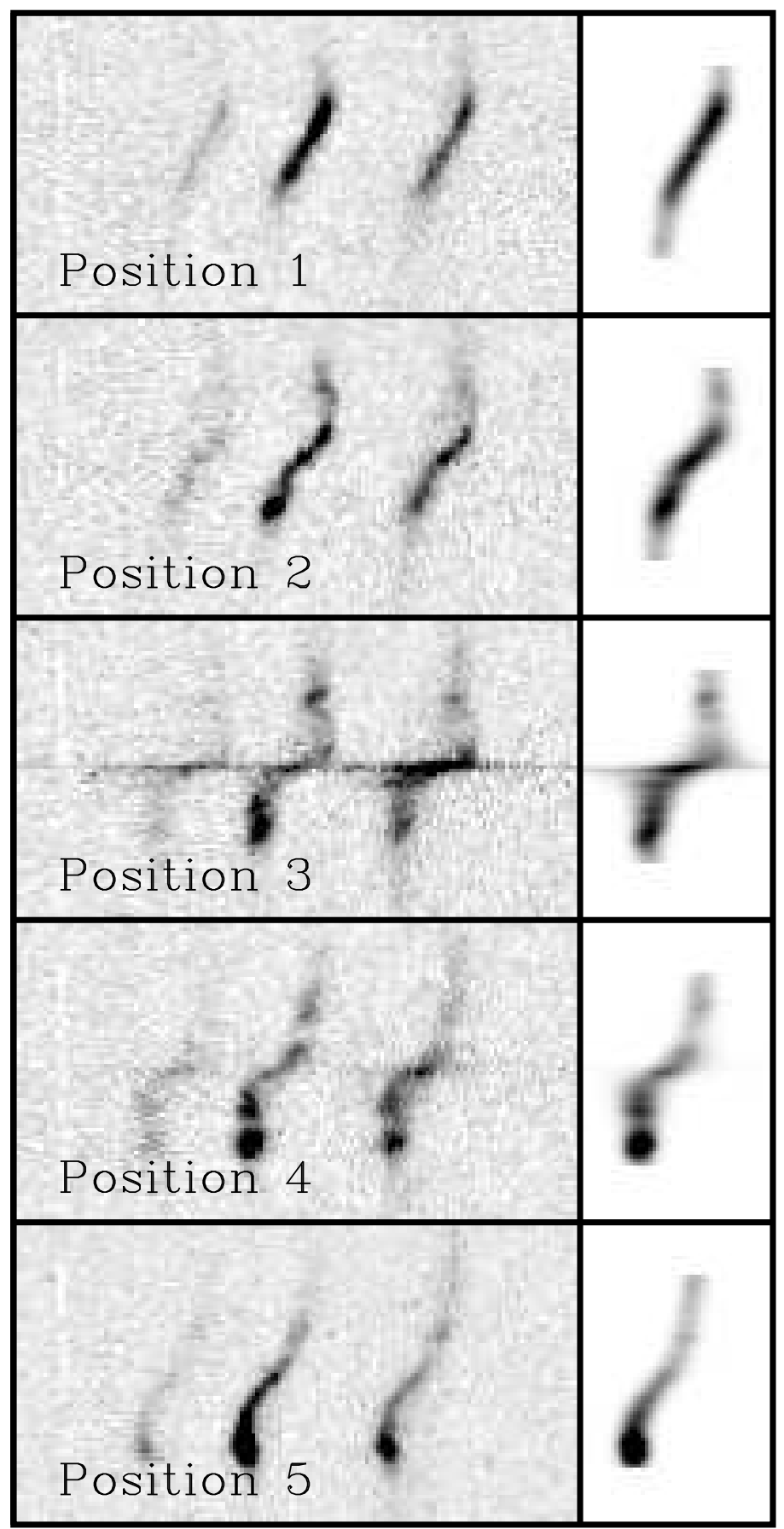

Fig. 4.- Left panels: Portions of the geometrically rectified two-dimensional STIS spectra showing the $\mathrm{H} \alpha+[\mathrm{N} \mathrm{II}]$ emission blend. The spatial axis is vertical with the same orientation as in Figure 1 , and wavelength increases to the right. Continuum emission from the galaxy bulge has been subtracted. Each box has dimensions $4^{\prime \prime} 0$ in the spatial direction and $83 \AA$ in the dispersion direction. Right panels: The two-dimensional synthetic spectra for each slit position for the bestfitting disk model. 


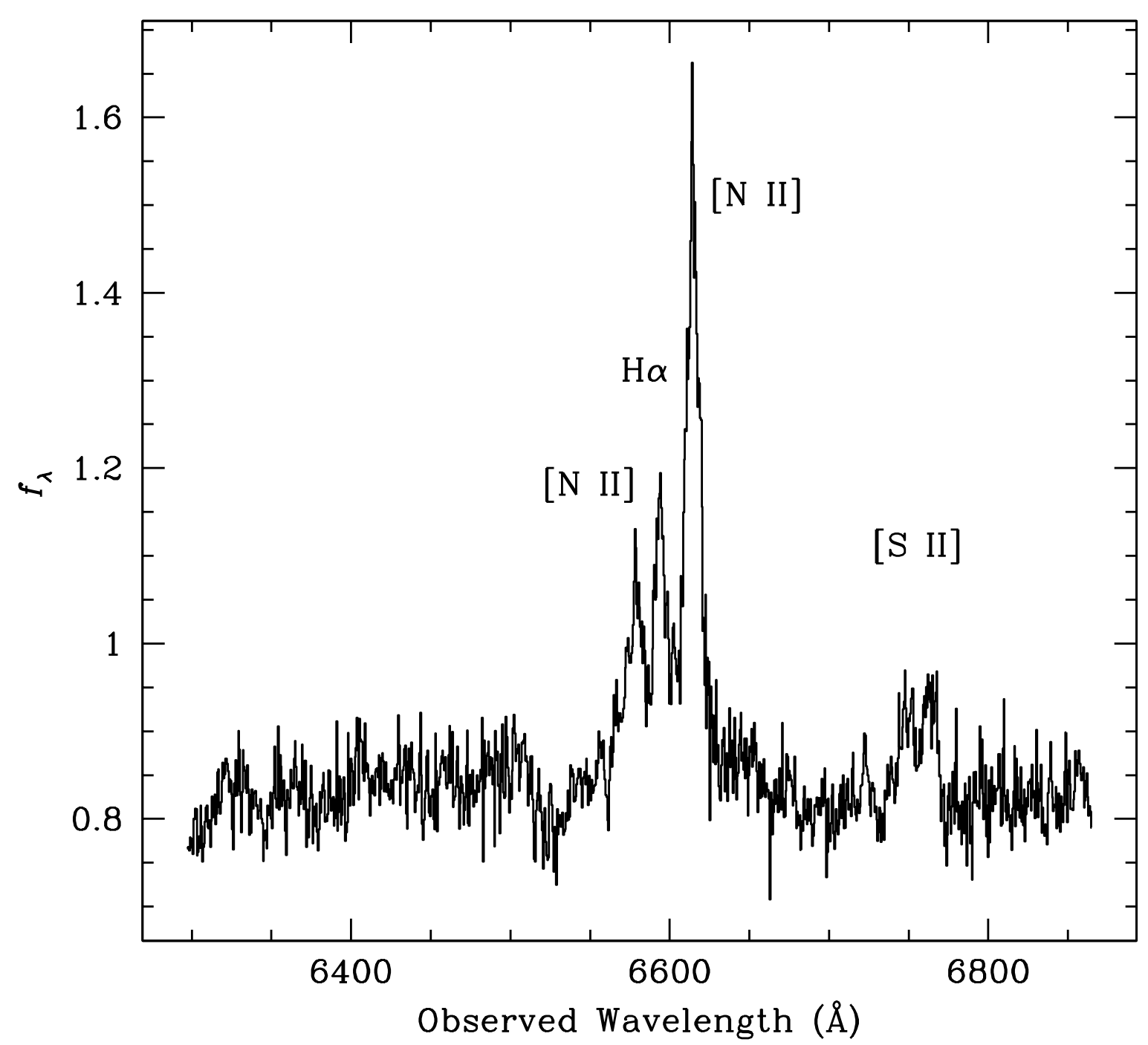

Fig. 5.- The nuclear spectrum of NGC 3245, from slit position 3. This extraction is the sum of five CCD rows ( 0 '! 25 total) centered on the nucleus, with no background subtraction applied. The vertical axis is in units of $10^{-16} \mathrm{erg} \mathrm{cm}^{-2} \mathrm{~s}^{-1} \AA^{-1}$. 


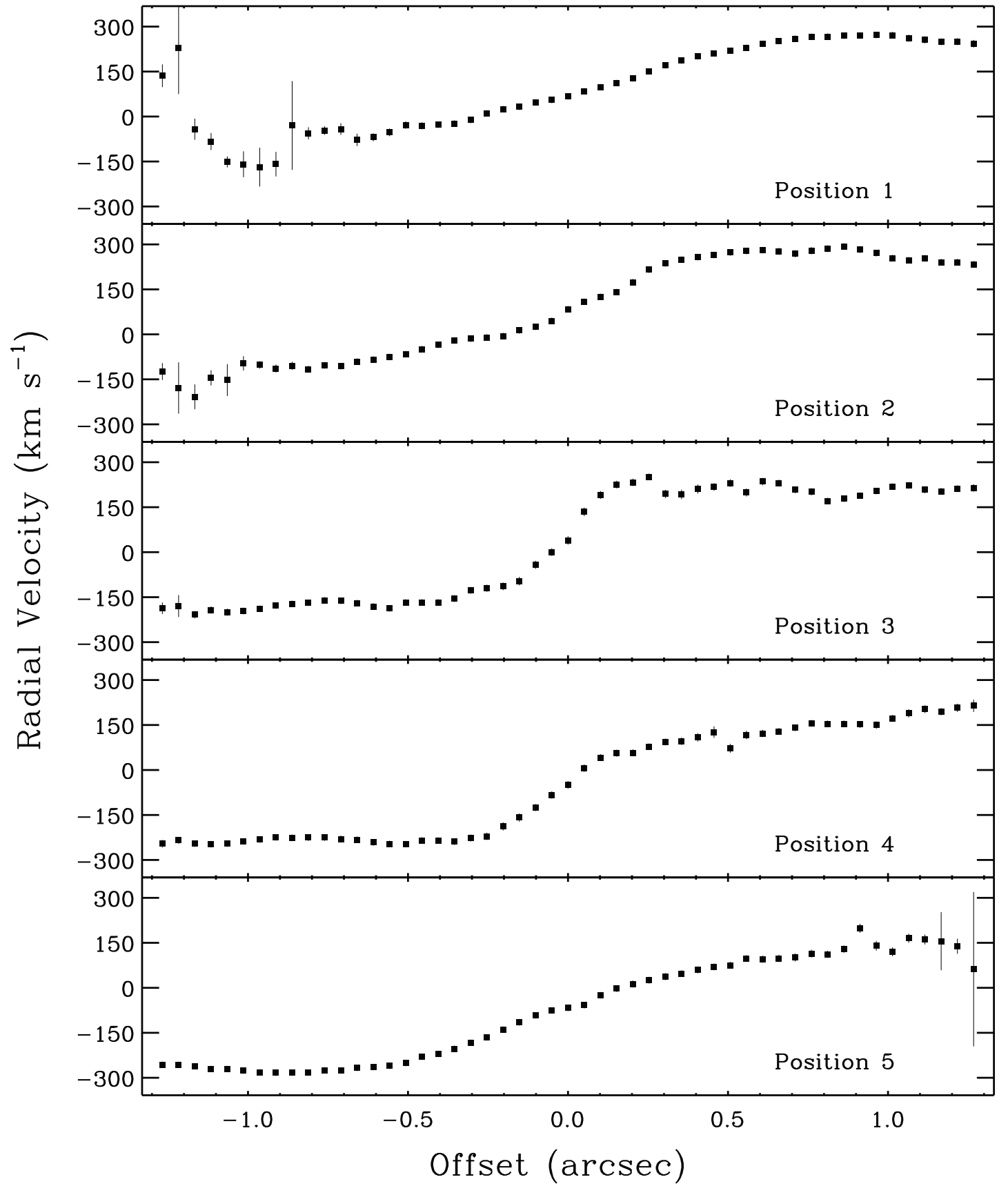

Fig. 6. - Velocity curves for the five slit positions. The zeropoint of the radial velocity scale is the galaxy's systemic velocity of $1388 \mathrm{~km} \mathrm{~s}^{-1}$. The left side of this plot corresponds to the bottom of the slit (i.e., the northern side of the disk) in Figure 1. 


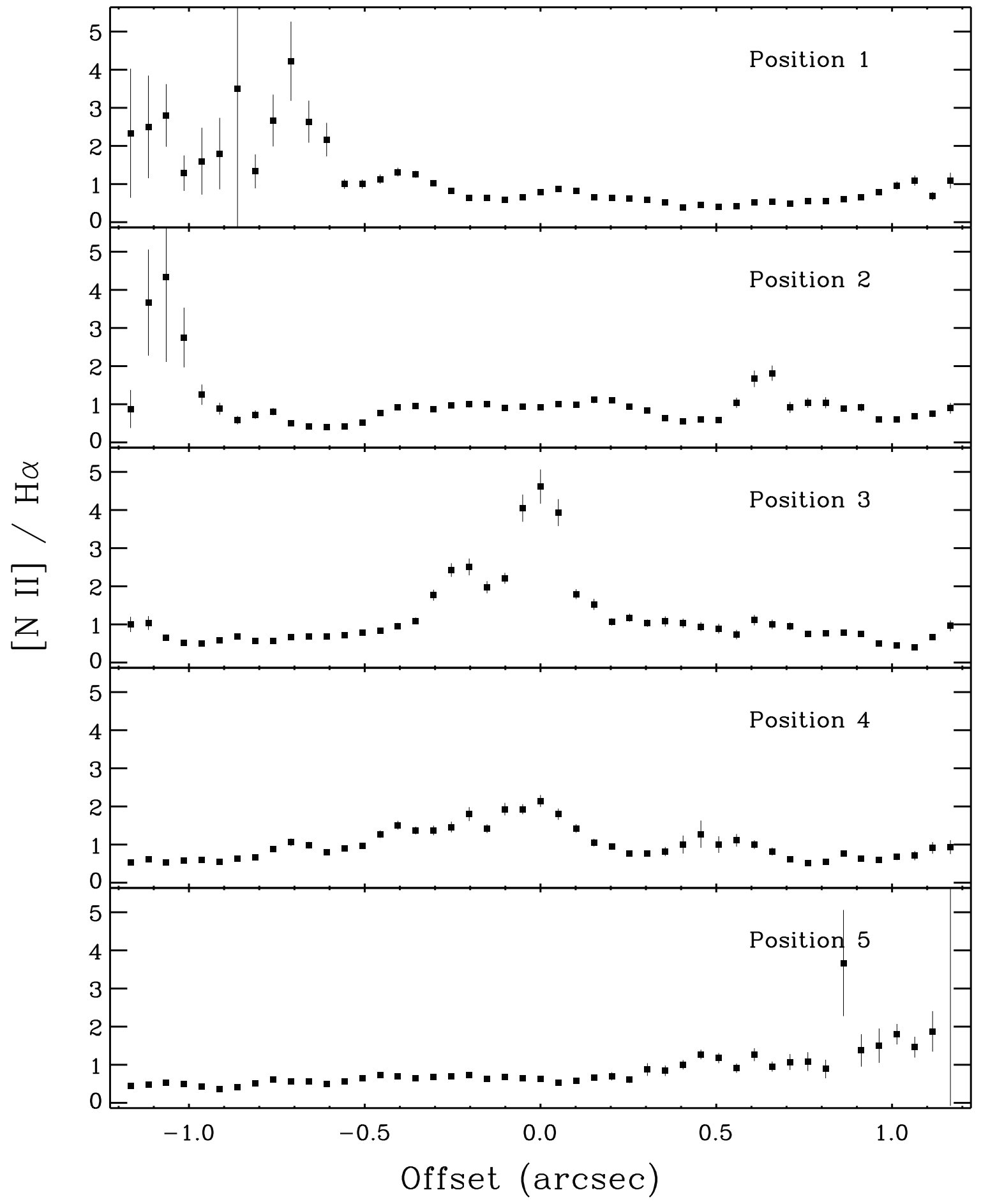

Fig. 7.- The [N II] $\lambda 6584 / \mathrm{H} \alpha$ emission-line intensity ratio at each position in the STIS spectra. The broad component of $\mathrm{H} \alpha$ (detected only in the innermost three pixels of Position 3) is not included. 


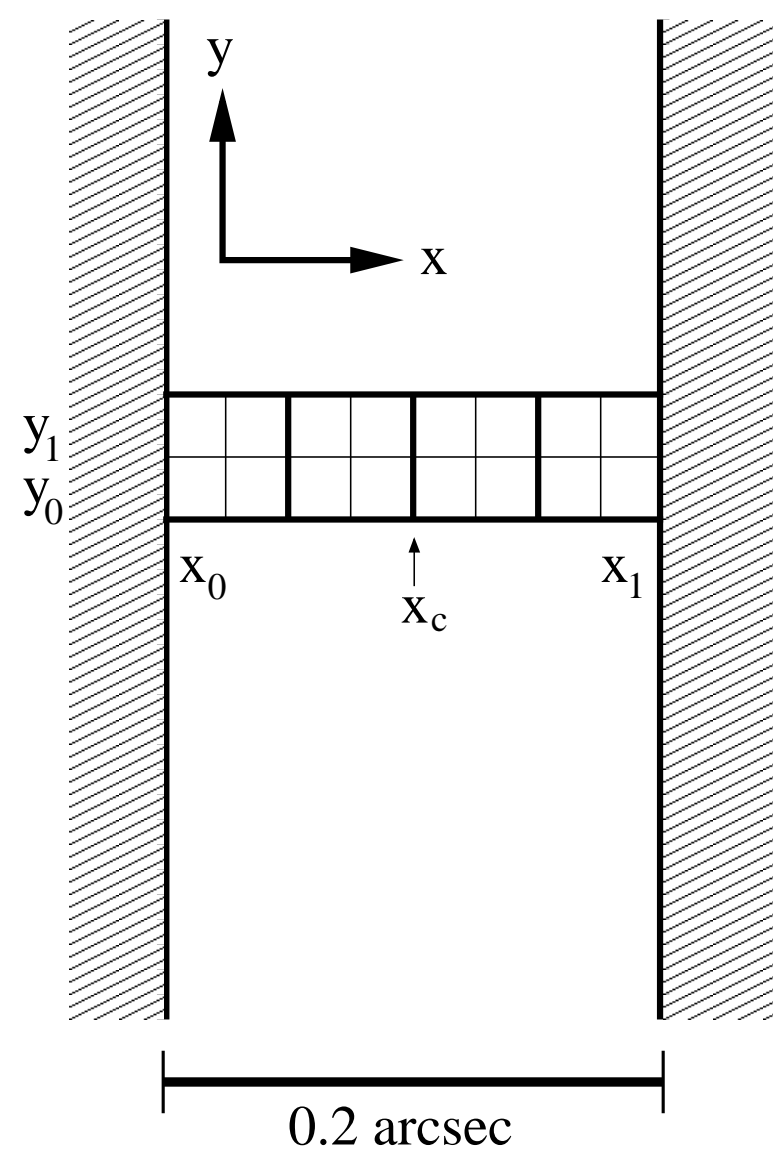

Fig. 8.- Diagram illustrating the slit geometry used in the line-profile calculations (equation 4). For a given STIS CCD row, the predicted line profile is computed by summing the contributions of light entering the slit through a $4 s \times s$ grid of subpixel elements, where $s$ is the subsampling factor. The light entering each subpixel element includes contributions from each point in the model velocity field, weighted by the $\mathrm{H} \alpha+[\mathrm{N} \mathrm{II}]$ surface brightness and the PSF. The slit midpoint in the $x$ direction is located at $x_{c}$. The diagram shows the case $s=2$. 


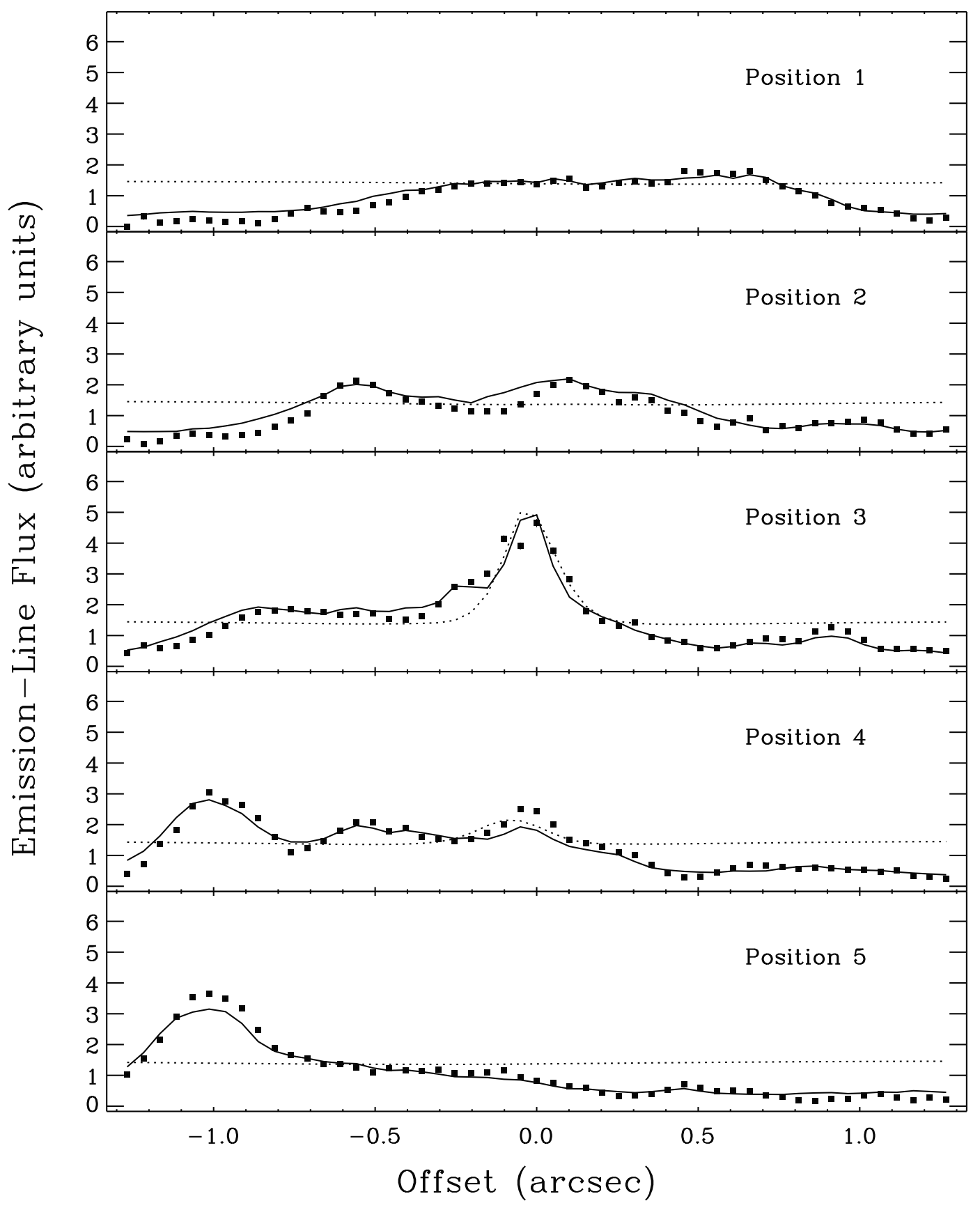

Fig. 9.- The total $\mathrm{H} \alpha+[\mathrm{N} \mathrm{II}]$ emission-line flux measured at each position in the STIS spectra. Solid curves: Model results using the continuum-subtracted, deconvolved WFPC2 F658N image as the $\mathrm{H} \alpha+[\mathrm{N}$ II] surface brightness. A single scaling factor has been applied in order to match the median values of the STIS data and the model curves. Dotted curves: Results of calculations based on the exponential surface-brightness model. 


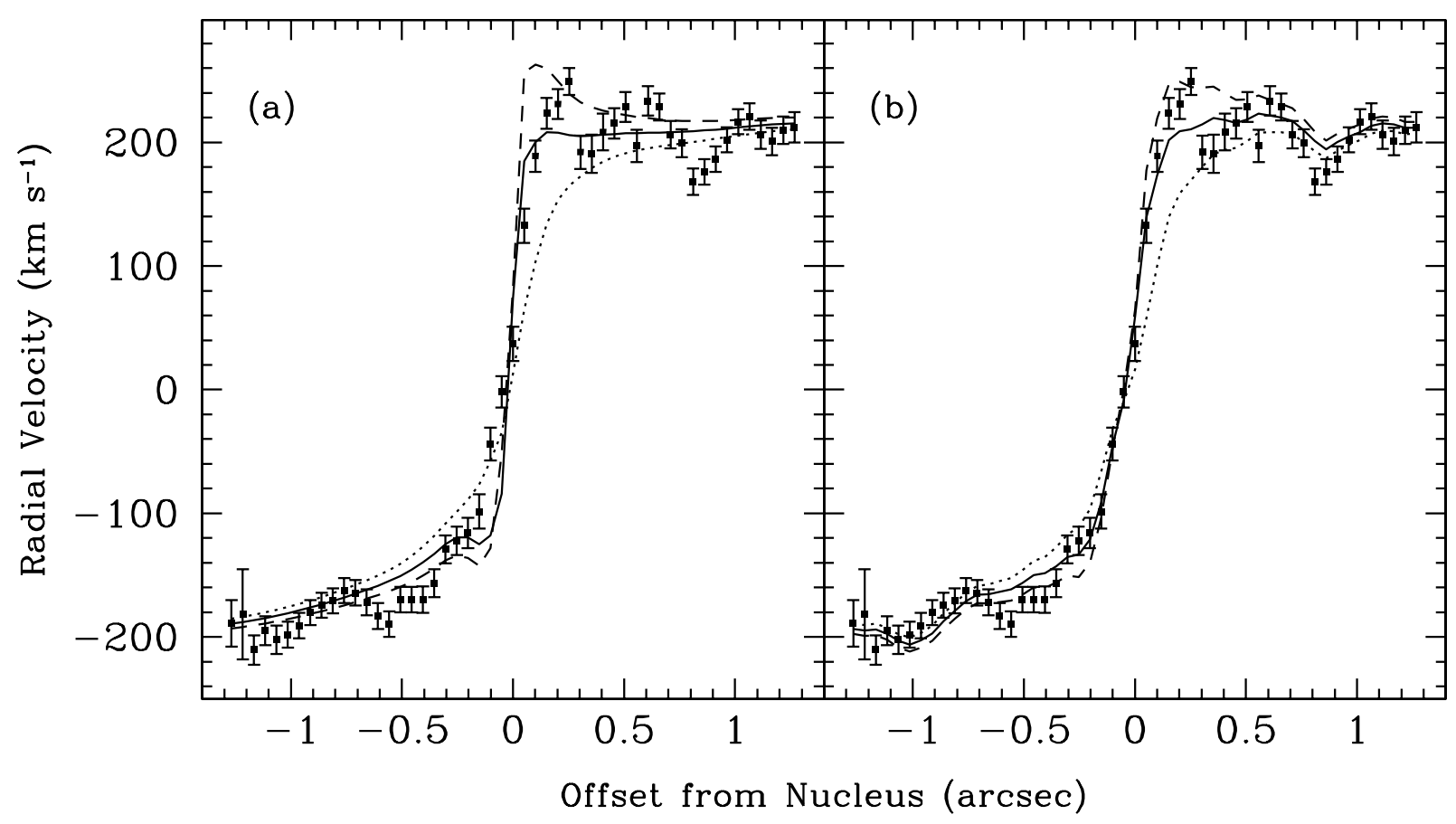

Fig. 10. - Radial velocity curves for slit position 3. (a) Models computed using the exponential model to approximate the $\mathrm{H} \alpha+[\mathrm{N} \mathrm{II}]$ surface brightness. (b) The same models, but computed using the $\mathrm{H} \alpha+[\mathrm{N}$ II] surface brightness measured from the continuum-subtracted, deconvolved WFPC2 F658N image. BH masses are 0 (dotted line), $2 \times 10^{8} M_{\odot}$ (solid curve), and $4 \times 10^{8} M_{\odot}($ dashed curve). The models are computed for $\Upsilon=3.5 \Upsilon_{\odot}$ to illustrate the fit to the outer portions of the disk, but the inner $r=0.5$ would be better fit with $\Upsilon$ in the range $3.6-3.8 \Upsilon_{\odot}$. 


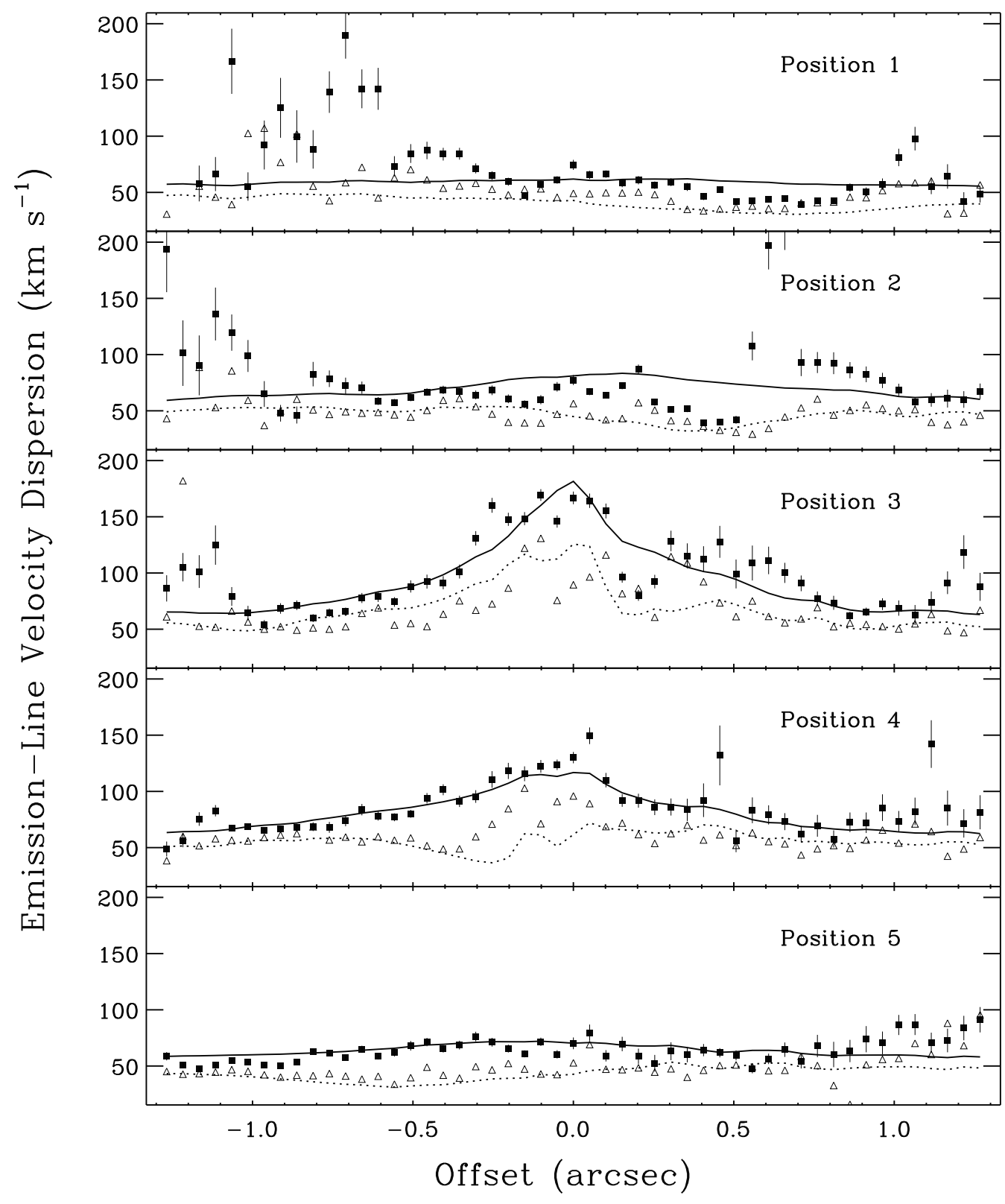

Fig. 11. - Velocity dispersions of [N II] (filled squares) and $\mathrm{H} \alpha$ (open triangles) at each position in the STIS spectra. For clarity, error bars on the $\mathrm{H} \alpha$ velocity dispersions have been omitted. Dotted curves: Results of model calculations for a dynamically cold disk with zero intrinsic linewidth. In these models, the only contributions to the predicted linewidths are rotational and instrumental broadening. Solid curves: Model results incorporating the intrinsic velocity dispersion in the disk, with parameters set to fit the $[\mathrm{N}$ II] data. Regions outside the nucleus showing very high velocity dispersions (at the left side of positions 1 and 2) are the result of low $\mathrm{S} / \mathrm{N}$ at these positions; since velocity dispersion is always positive, random errors at very low $\mathrm{S} / \mathrm{N}$ will bias the measurements toward high values of $\sigma$. 


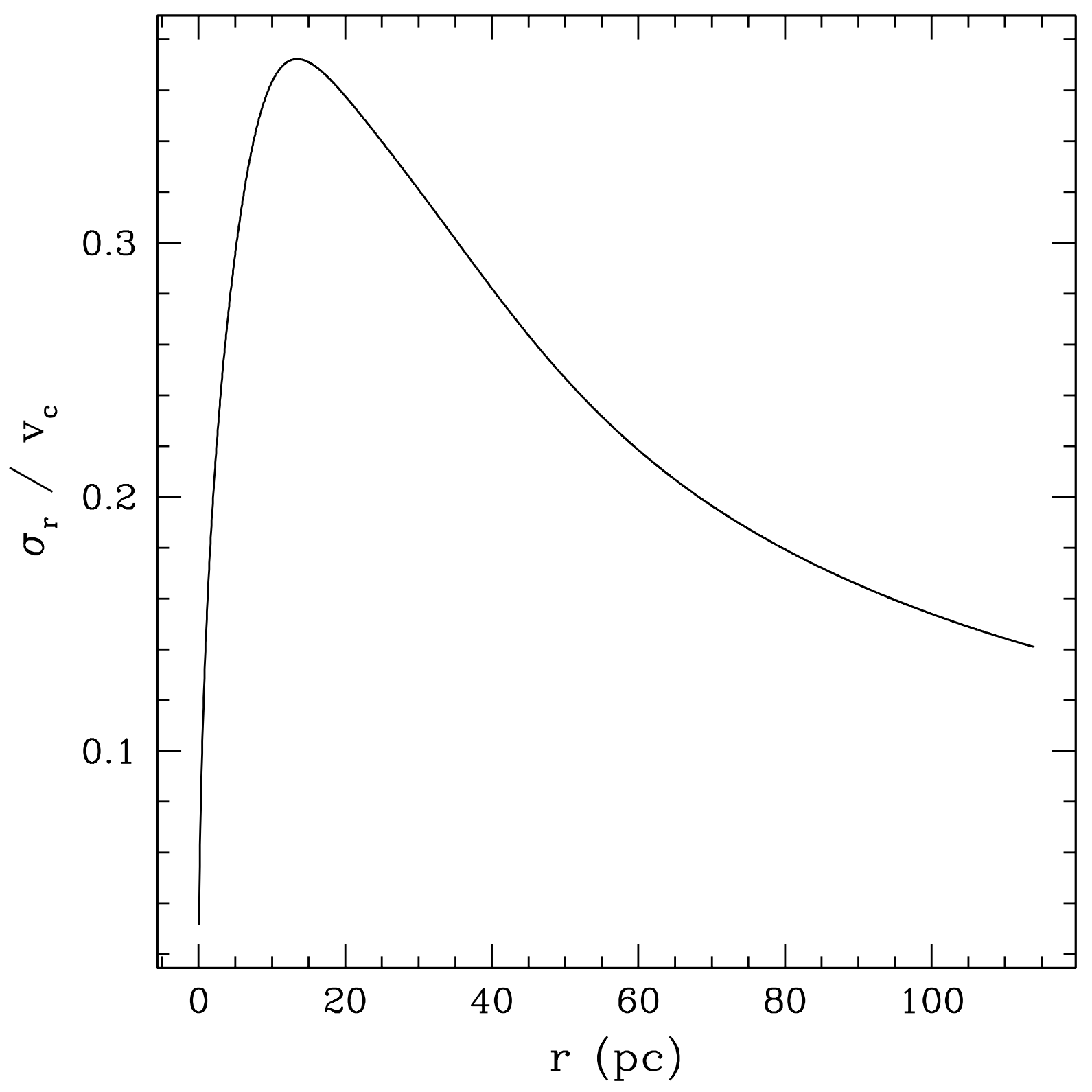

Fig. 12.- The ratio $\sigma_{r} / v_{c}$ for the best-fitting model to the NGC 3245 data. 


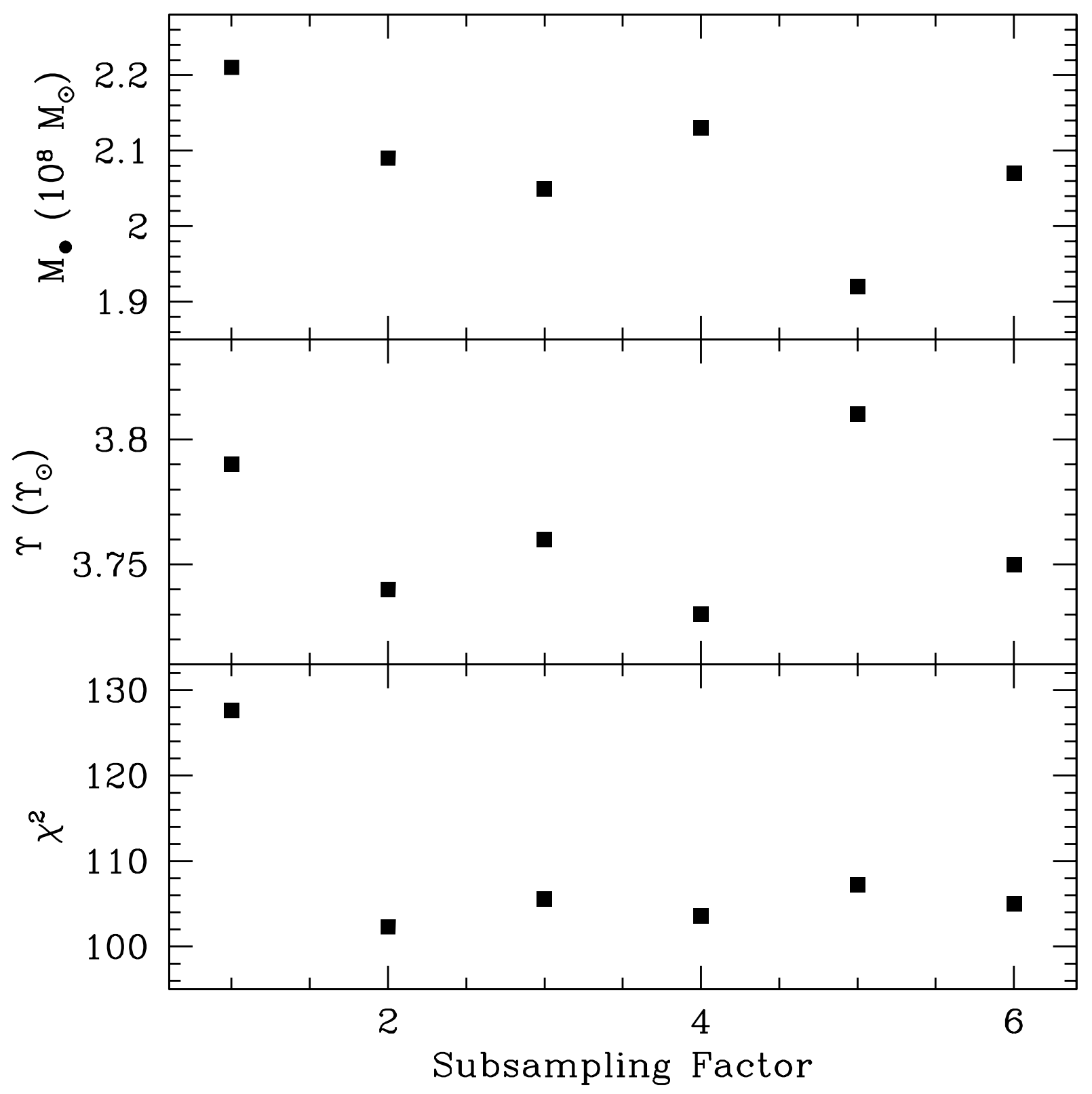

Fig. 13.- Results of model fits for different values of the subsampling factor $s$. The quantities $M_{\bullet}$, $\Upsilon, i, \theta$, and $v_{\text {sys }}$ were allowed to float as free parameters. These models were calculated with no asymmetric drift correction, and with $r_{\text {fit }}=0$ '.5. 


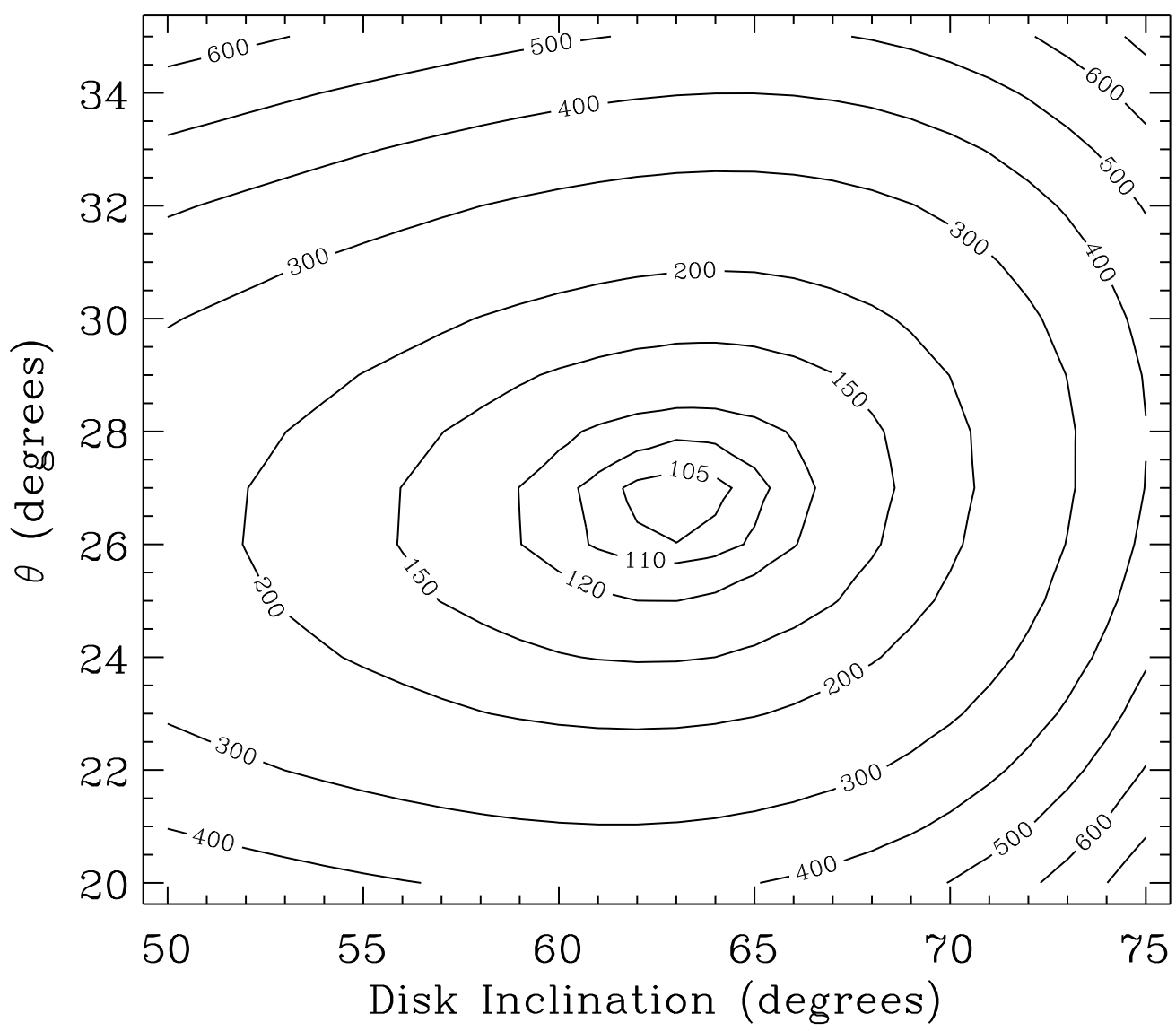

Fig. 14.- Contours of constant $\chi^{2}$ for models with varying values of disk inclination $i$ and offset angle $\theta$ between the slit P.A. and the disk major axis. Models were calculated with no asymmetric drift correction, $s=2$, and $r_{\text {fit }}=0.5$. At each grid point, $M_{\bullet}, \Upsilon$, and $v_{\text {sys }}$ were allowed to float freely. 


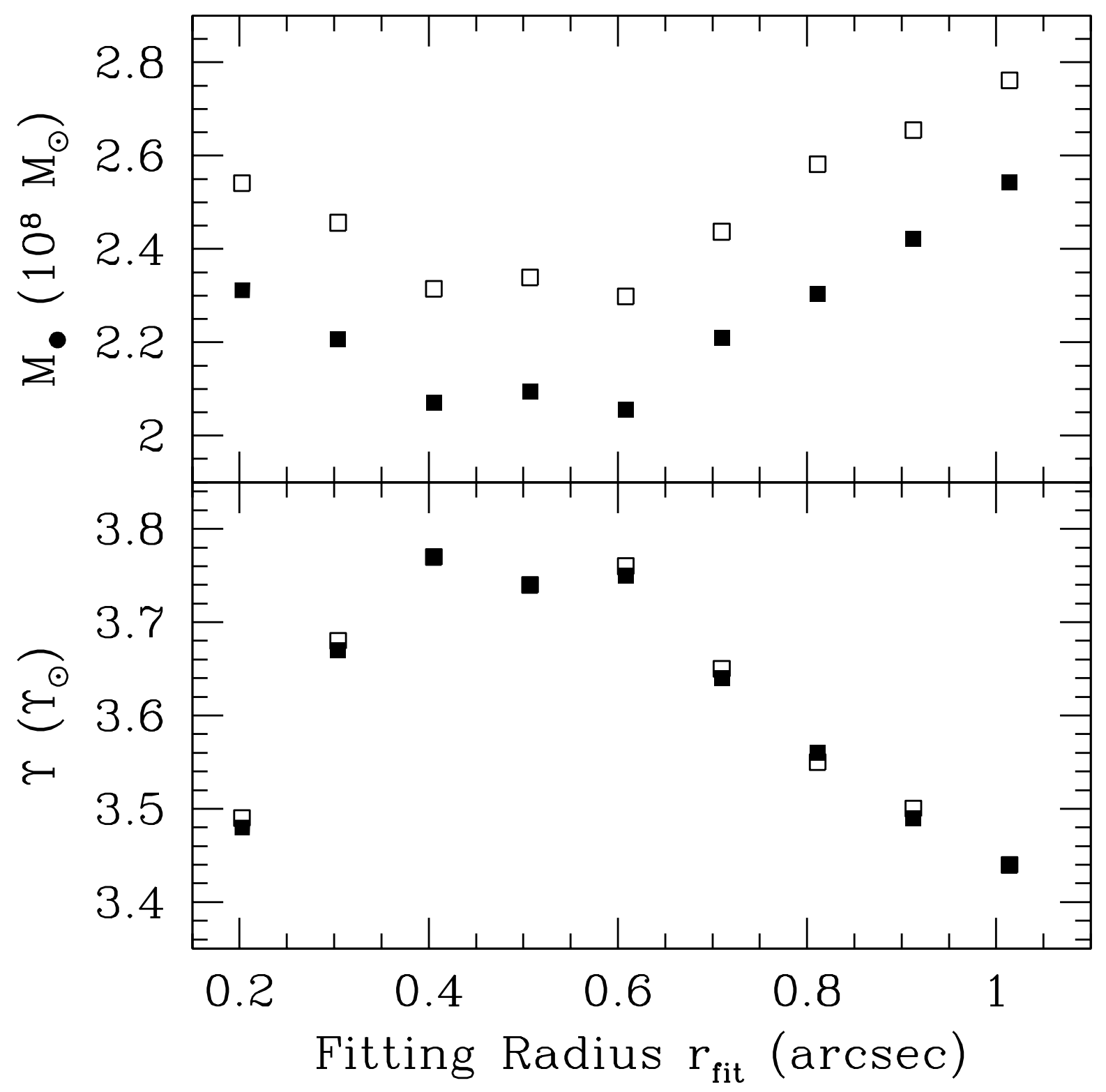

Fig. 15. - Results of model fits for different values of $r_{\text {fit }}$. In each calculation, $M_{\bullet}$ and $\Upsilon$ are allowed to float while $i$ and $\theta$ are fixed at $63^{\circ}$ and $27^{\circ}$, respectively. Models with and without the asymmetric drift correction are indicated by open and filled squares, respectively. 


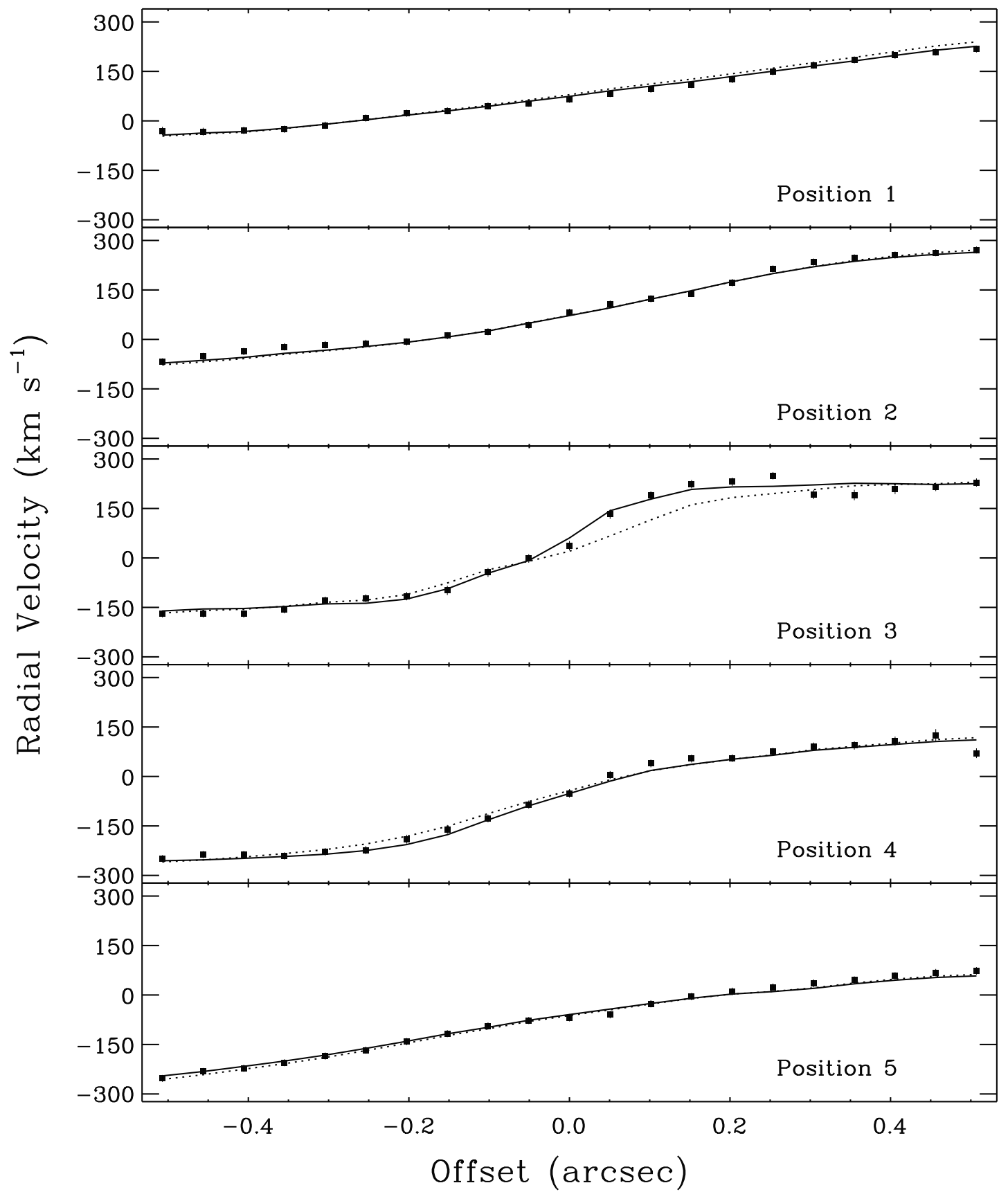

Fig. 16. - Comparison of the $\mathrm{H} \alpha+[\mathrm{N}$ II] radial velocities measured from the STIS data with model predictions. Solid curve: Best-fitting model with $r_{\text {fit }}=0$. . 5 . This model has $M_{\bullet}=2.09 \times 10^{8} M_{\odot}$ and $\Upsilon=3.74 \Upsilon_{\odot}$. Dashed curve: The best-fitting model for the case $M_{\bullet}=0$ and $r_{\text {fit }}=0.5$. For this model, $\Upsilon=4.61 \Upsilon \odot$. 


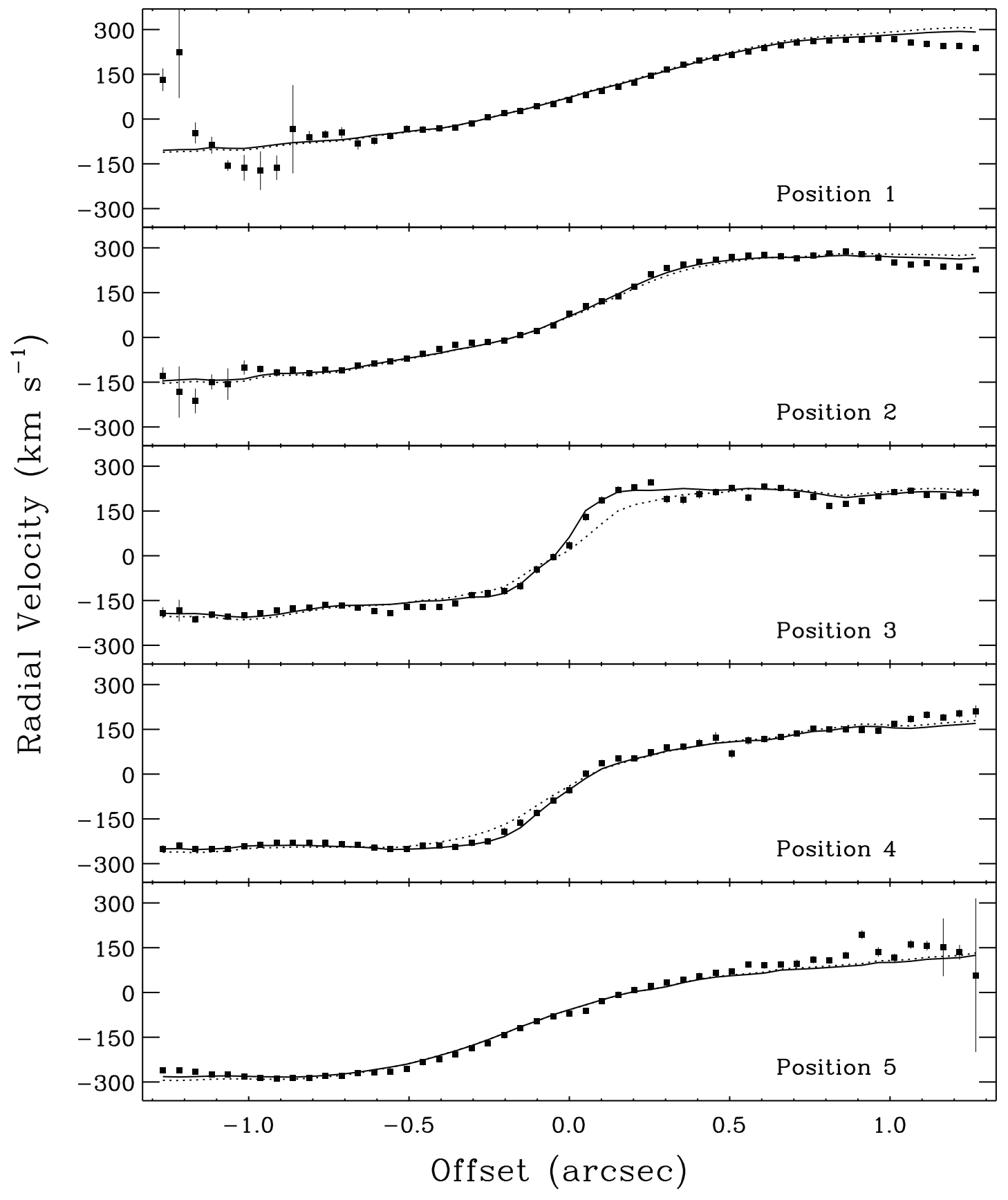

Fig. 17.- Comparison of the $\mathrm{H} \alpha+[\mathrm{N}$ II $]$ radial velocities measured from the STIS data with model predictions. Solid curve: Best-fitting model for the case $r_{\text {fit }}=1^{\prime \prime}$. 0 . This model has $M_{\bullet}=$ $2.54 \times 10^{8} M_{\odot}$ and $\Upsilon=3.44 \Upsilon_{\odot}$. Dotted curve: Best-fitting model for the case $M_{\bullet}=0$ and $r_{\text {fit }}=$ 1".0. This model has $\Upsilon=4.04 \Upsilon_{\odot}$. 


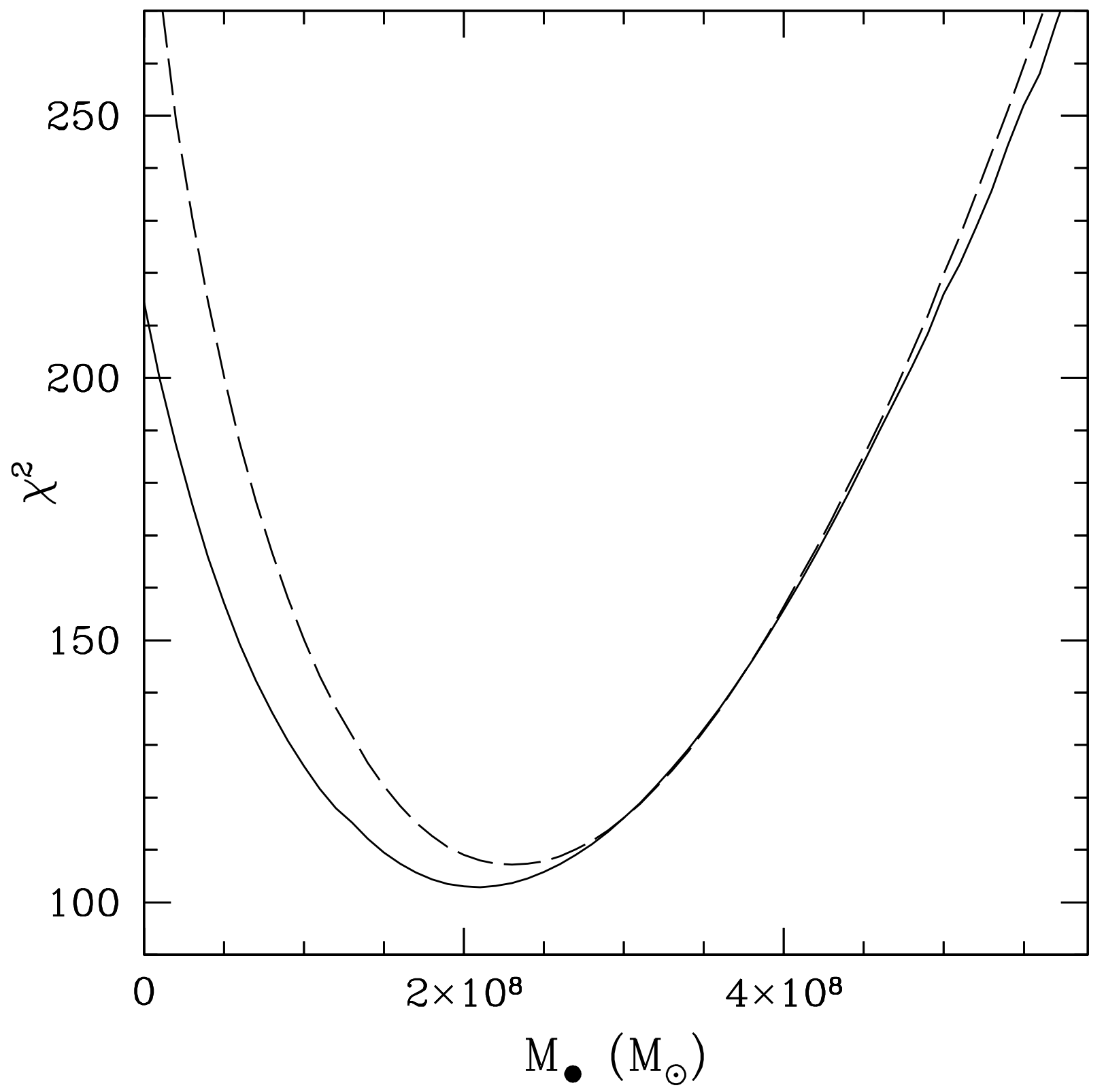

Fig. 18. - Results of disk-model fits for $r_{\text {fit }}=0.5$ and $s=2$. Each model was calculated by fixing $M_{\bullet}$ and then optimizing $\Upsilon, i, \theta$, and $v_{\text {sys }}$ to minimize $\chi^{2}$ for the given value of $M_{\bullet}$. Solid curve: Models without asymmetric drift correction. Dashed curve: Models including the asymmetric drift correction. 


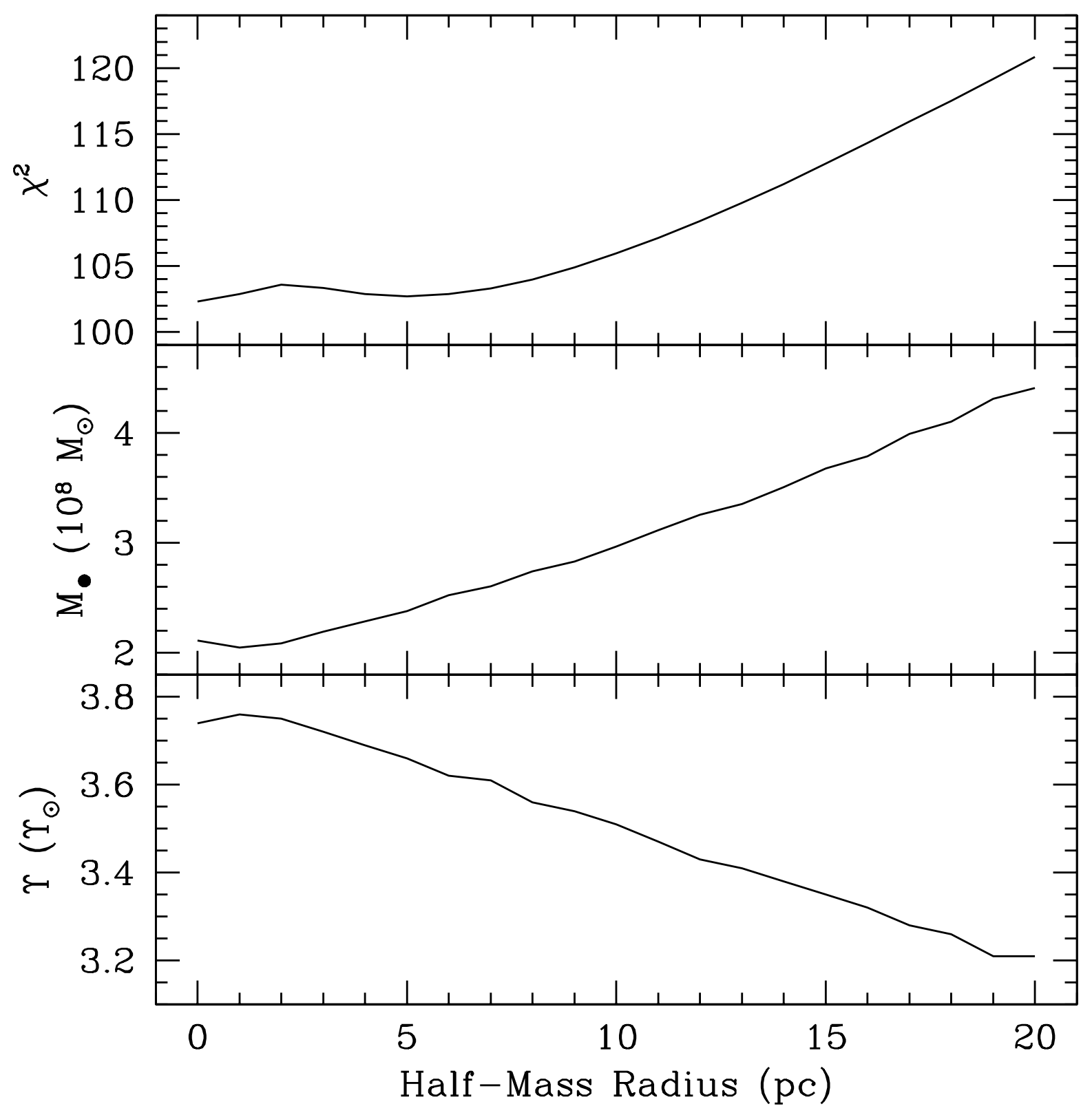

Fig. 19. - Results of model fits for models with a spatially extended central dark mass, modeled as a Plummer sphere. For each fixed value of $r_{1 / 2}$, models were calculated with the quantities $M_{\bullet}$, $\Upsilon$, and $v_{\text {sys }}$ as free parameters. 


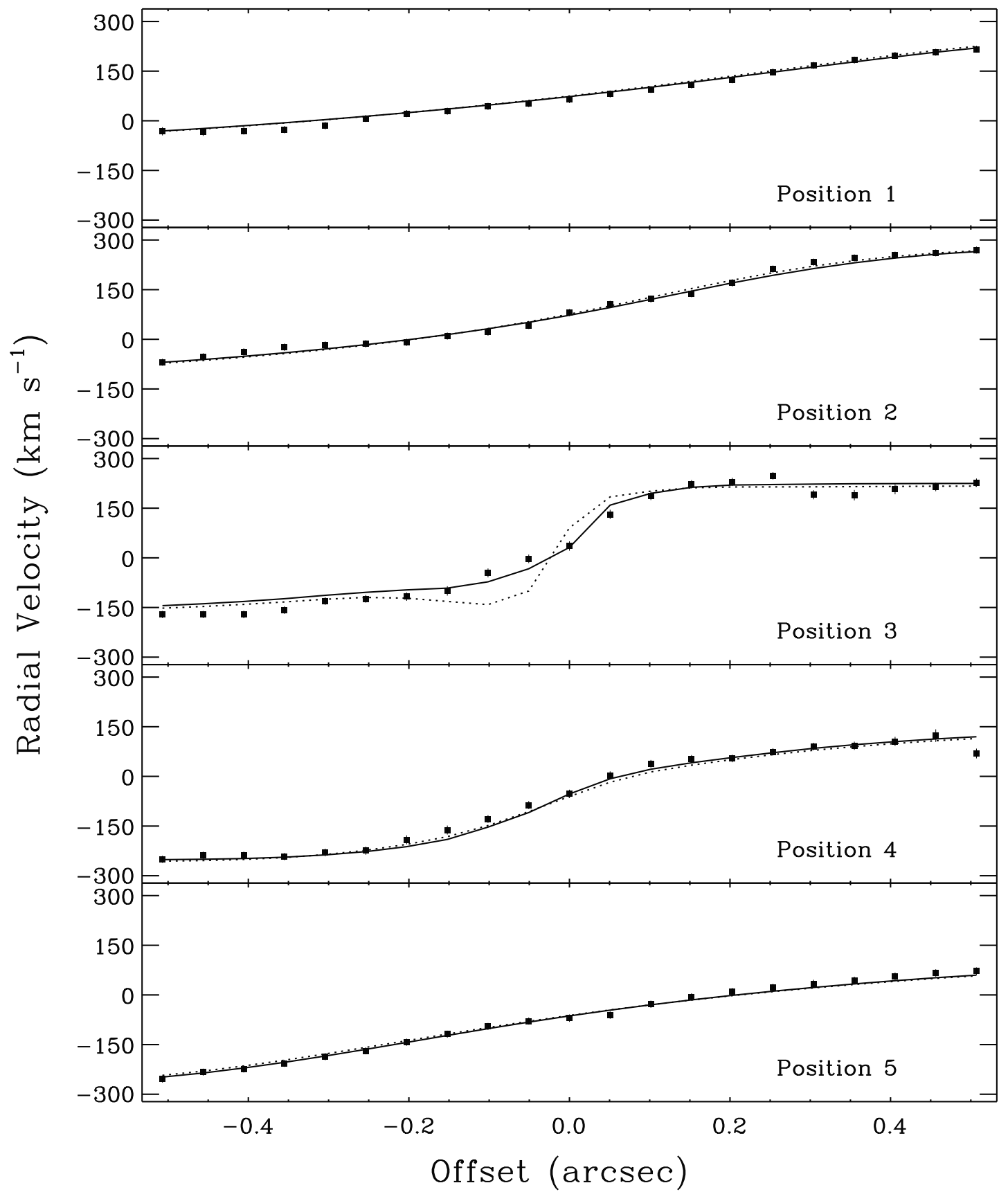

Fig. 20.- Result of model calculations using the exponential surface-brightness model. Solid curve: Results for $s=1$. Dotted curve: Results for $s=4$. Both models are computed with Tiny Tim PSFs and with instrumental velocity shifts; parameters are given in Table 2. 